\title{
APPROXIMATIONS OF SOBOLEV NORMS IN CARNOT GROUPS
}

\author{
DAVIDE BARBIERI
}

\begin{abstract}
This paper deals with a notion of Sobolev space $W^{1, p}$ introduced by J.Bourgain, H.Brezis and P.Mironescu by means of a seminorm involving local averages of finite differences. This seminorm was subsequently used by A.Ponce to obtain a Poincaré-type inequality. The main results that we present are a generalization of these two works to a non-Euclidean setting, namely that of Carnot groups. We show that the seminorm expressd in terms of the intrinsic distance is equivalent to the $L^{p}$ norm of the intrinsic gradient, and provide a Poincaré-type inequality on Carnot groups by means of a constructive approach which relies on one-dimensional estimates. Self-improving properties are also studied for some cases of interest.
\end{abstract}

\section{INTRODUCTION}

This paper is based on a sequence of works by J.Bourgain, H.Brezis and P.Mironescu 3, H.Brezis 4] and A.Ponce 25 concerning a peculiar approach to Sobolev spaces $W^{1, p}$, which allows to obtain Sobolev norms in terms of integral averages of finite differences, suitably localized by means of radial mollifiers.

This technique provides finite scale approximations of first derivatives which can be used to define Sobolev $(1, p)$ norms that are equivalent to the ordinary ones and reduce to them by letting this scale become infinitely small.

We developed a generalization of the main results concerning this technique to the setting of Carnot groups, which represent a class of nonEuclidean metric spaces widely studied in the context of degenerate PDE's, complex analysis and control theory, lying at the basis of the so-called subRiemannian geometry.

A key result of the cited approximating technique is expressed by 3 , Corollary 1], where it is shown that for $f \in W^{1, p}(\Omega), \Omega$ being a smooth bounded domain in $\mathbb{R}^{N}$, it holds

$$
\int_{\Omega} \frac{|f(y)-f(x)|^{p}}{|y-x|^{p}} \rho_{n}(|y-x|) d y \stackrel{n \rightarrow \infty}{\longrightarrow} K|\nabla f(x)|^{p} \quad \text { in } \quad L^{1}(\Omega)
$$

where $K=K(p, N)$ and $\rho_{n}$ are radial mollifiers: nonnegative functions such that

$$
\int \rho_{n}(|x|) d x=1, \text { and } \lim _{n \rightarrow \infty} \int_{\delta}^{\infty} \rho_{n}(r) r^{N-1} d r=0 \quad \forall \delta>0 .
$$

The author has been supported by University of Bologna, Université de Cergy-Pontoise, Université Franco-Italienne Programme Vinci, and by European Project GALA.. 
Various applications of this method can be found in the cited references, and among them there is the availability of a Poincaré-type inequality, see [25], which makes use of the described integral approximation: for $f \in L^{p}(\Omega)$

$$
\int_{\Omega}\left|f(x)-f_{\Omega}\right|^{p} d x \leq C \int_{\Omega} \int_{\Omega} \frac{|f(y)-f(x)|^{p}}{|y-x|^{p}} \rho_{n}(|y-x|) d y d x \quad \forall n>n_{0}
$$

$f_{\Omega}$ being the average of $f$ on $\Omega, C$ a constant which depends on $p, N$ and on the constant of the ordinary Poincaré inequality and $n_{0}=n_{0}(C, \Omega)$.

In order to extend to Carnot groups this finite differences approach to Sobolev norms, originally exploited in a purely Euclidean setting, we could apply techniques similar to those used in [4] by making use of the differentiable structure introduced in 24] (see also [2, [6, [9]) and of the homogeneity of the space. In particular our proof shows how the essential properties needed to obtain results as sharp as in the Euclidean case can be obtained in the case of Carnot groups.

For what concerns the Poincaré inequality, our proof is essentially different from the one given in [25]: due to the non-Euclidean composition law of the group, the original procedure cannot be applied, so we provided a new constructive proof that relies on a one-dimensional inequality. The main advantage of this approach consists of the fact that it shows quantitatively the relationship between the size of the bounded domain on which the mean oscillation of the function is evaluated and the scale at which the gradient is approximated by finite differences.

Carnot groups [2], 26], 28], [7, [15], 8] are Lie groups endowed with a homogeneous structure given by a family of anisotropic dilations. The Lie algebras of Carnot groups are stratified: a linear subspace sufficient to generate the whole algebra can be defined from the commutation relations, or equivalently from the group composition law. This subspace is generally called the horizontal layer and, thanks to the Chow connectivity theorem, it defines an intrinsic metric structure, called the Carnot-Carathéodory distance, which is given by the infimum length among all the integral curves of horizontal vector fields connecting two points. Other equivalent metric structures on Carnot groups are those induced by a so-called homogeneous norm by making use of group operations. The homogeneous structure induces another peculiarity of Carnot groups: the Hausdorff dimension is in general higher than the topological dimension. This phenomenon is due to the different scalings of the directions, which contribute to the measure in a non uniform way; from the algebra point of view, it reflects the fact that some directions are "heavier" than others, because they belong to higher layers.

In what follows, $\mathbb{G}$ will indicate a Lie group, and $\mathfrak{g}$ a Lie algebra. By Lie algebra $\mathfrak{g}$ of a Lie group $\mathbb{G}$, we will mean the usual linear space of leftinvariant vector fields on $\mathbb{G}$, with the Lie bracket given by the commutator: $([X, Y] f)(x) \doteq(X(Y f))(x)-(Y(X f))(x), \quad X, Y \in \mathfrak{g}$. The identification of $\mathfrak{g}$ with $T_{\mathfrak{e}} \mathbb{G}$, the tangent space at the identity $\mathfrak{e}$ of $\mathbb{G}$, will also be assumed. As a global reference on this topic, we suggest [27]. Here we will only recall briefly the notion of exponential map for $\mathbb{G}$ a Lie group and $\mathfrak{g}$ its Lie algebra: 
let $\gamma_{X, x}(t)$ the integral curve of $X \in \mathfrak{g}$ passing through $x \in \mathbb{G}$

$$
\begin{aligned}
\gamma_{X, x}^{\prime}(t) & =X\left(\gamma_{X, x}(t)\right) \\
\gamma_{X, x}(0) & =x .
\end{aligned}
$$

The exponential map is then defined as

$$
\begin{aligned}
\exp : \quad \mathfrak{g} & \longrightarrow \mathbb{G} \\
X & \longrightarrow \exp (X) \doteq \gamma_{X, \mathfrak{e}}(1)
\end{aligned}
$$

and it is a local diffeomorphism of a neighborhood of $0 \in \mathfrak{g}$ to a neighborhood of $\mathfrak{e} \in \mathbb{G}$ :

$$
d \exp (0)=\mathbb{I}_{\mathfrak{g}}
$$

which extends to a global diffeomorphism if $\mathbb{G}$ is simply connected, so it allows to construct systems of coordinates on $\mathbb{G}$ given a basis $\left\{X_{j}\right\}_{j=1}^{N}$ of g. In particular an element $x \in \mathbb{G}$ is said to have canonical coordinates $\left(x_{1}, \ldots, x_{N}\right) \in \mathbb{R}^{N}$ if $x=\exp \left(x_{1} X_{1}+\cdots+x_{N} X_{N}\right)$.

\section{Acknowledgments}

This paper is a part of author's $\mathrm{PhD}$ thesis, written under the supervision of Thierry Coulhon and Bruno Franchi, to whom the author is particularly indebted for their careful directions. The author thanks also Augusto Ponce and Francesco Serra Cassano for helpful discussions.

\section{Carnot Groups}

Definition 2.1 (Carnot groups). A Carnot group $\mathbb{G}$ is a simply connected Lie group with a stratified Lie algebra $\mathfrak{g}$ i.e. a nilpotent Lie algebra that can be decomposed into a direct sum of linear subspaces called layers, $\left\{W_{j}\right\}_{j=1}^{k}$, in such a way that the first layer generates the whole algebra:

$$
\mathfrak{g}=\bigoplus_{j=1}^{k} W_{j}, \quad W_{j+1}=\left[W_{1}, W_{j}\right] \forall j<k, \quad W_{j}=0 \quad \forall j>k .
$$

The positive integer $k$ is called the step of $\mathfrak{g}$.

We now state some basic properties of such groups.

Dilations: given a stratified Lie algebra $\mathfrak{g}$, it is possible to define algebra dilations as a 1-parameter group $\left\{\Delta_{\lambda}\right\}_{\lambda>0}$ of automorphisms of $\mathfrak{g}$ which act on layers as

$$
\Delta_{\lambda}(X)=\lambda^{j} X \quad \forall X \in W_{j} .
$$

Algebra dilations induce on $\mathbb{G}$, through exponential mapping, a 1-parameter group of automorphisms of $\mathbb{G}$ called group dilations $\left\{\delta_{\lambda}\right\}_{\lambda>0}$ :

$$
\delta_{\lambda}\left(\delta_{\mu}(x)\right)=\delta_{\lambda \mu}(x), \delta_{\lambda}(x \cdot y)=\delta_{\lambda}(x) \cdot \delta_{\lambda}(y) \quad \forall x, y \in \mathbb{G} \quad \forall \lambda, \mu>0 .
$$

The quantity $Q=\sum j \operatorname{dim}\left(W_{j}\right)$ is called the homogeneous dimension of $\mathbb{G}$, which equals its Hausdorff dimension as we will see in Corollary 2.11.

IDENTificAtion With $\mathbb{R}^{N}$ : since the exponential map is a global diffeomorphism, we can identify $\mathbb{G}$ with $\mathbb{R}^{N}$ endowed with the induced group law (which we shall denote by $\cdot$ ), $N$ being the linear dimension of $\mathfrak{g}$, by making use of canonical coordinates. If we set $m_{j}=\operatorname{dim}\left(W_{j}\right)$, then $\sum m_{j}=N$, which differs from $Q$ if $\mathfrak{g}$ has more than one layer. 
Given a basis $\left\{X_{1}, \ldots, X_{m_{1}}, X_{m_{1}+1}, \ldots, X_{m_{1}+m_{2}}, \ldots, X_{N}\right\}$ of $\mathfrak{g}$, grouped with respect to increasing layers, we can correspondingly order the canonical coordinates so that the action of group dilations reads

$$
\delta_{\lambda}\left(x_{1}, \ldots, x_{N}\right)=\left(\lambda x_{1}, \ldots, \lambda x_{m_{1}}, \lambda^{2} x_{m_{1}+1}, \ldots, \lambda^{2} x_{m_{1}+m_{2}}, \ldots, \lambda^{k} x_{N}\right) .
$$

Accordingly, we can group the canonical coordinates into distinct families $\left\{x_{1}, \ldots, x_{m_{1}}\right\}, \ldots,\left\{x_{m_{1}+\cdots+m_{k-1}}, \ldots, x_{N}\right\}$, and the ones corresponding to the $i$-th layer will be said to have weight $i$.

Because of the special role played by the first layer of $\mathfrak{g}$ (that defines the geometry of $\mathbb{G}$ ), it turns out to be convenient to distinguish the variables of weight 1 by putting

$$
x=\left(x^{\prime}, x^{\prime \prime}\right), \text { with } x^{\prime}=\left(x_{1}, \ldots, x_{m_{1}}\right) \text { and } x^{\prime \prime}=\left(x_{m_{1}+1}, \ldots, x_{N}\right)
$$

Vector operations on $\mathbb{R}^{N}$ will be performed as operations on $\mathbb{G}$ and in particular the identity $\mathfrak{e}$ of $\mathbb{G}$ is given by the vector $0 \in \mathbb{R}^{N}$.

Sub-RIEMANNIAN STRUCTURE: the first layer, or horizontal layer, of the algebra $\mathfrak{g}$ plays a central role in the geometry of Carnot groups. It can be identified with a linear subspace of the tangent space of $\mathbb{G}$ at the origin and defines, by left translations, a canonical subbundle $H \mathbb{G}$ of the tangent bundle $T \mathbb{G}$, whose fibers at points $x \in \mathbb{G}$ will be denoted by $H_{x} \mathbb{G}$. In particular $\mathbb{G}$ can be endowed with a sub-Riemannian metric: a scalar product $\langle., .\rangle_{X}$ defined on each horizontal fiber $H_{x} \mathbb{G}$ that makes the vector fields $X_{1}, \ldots, X_{m_{1}}$ orthonormal. The corresponding norm will be indicated as $|\cdot|_{X}$.

We say that a continuously differentiable curve $\gamma:[0, T] \rightarrow \mathbb{G}$ is horizontal if $\dot{\gamma}(t) \in H_{\gamma(t)} \mathbb{G}$ for all $0 \leq t \leq T$. Chow connectivity theorem asserts that any two points of $\mathbb{G}$ can be connected by a horizontal curve, settling the well-posedness of the following definition.

Definition 2.2 (Carnot-Carathéodory distance). The $C C$ distance is the intrinsic distance on Carnot groups, defined as the infimum sub-Riemannian length of horizontal curves connecting two points (see [9], 23], [15]):

$$
d_{X}(x, y) \doteq \inf _{\gamma \text { horizontal }} \int_{0}^{1}|\dot{\gamma}(t)|_{X} d t
$$

and we denote by $B_{X}(x, r)$ the $d_{X}$ metric balls.

Due to the left invariance of the vector fields, $d_{X}$ is left invariant, that is

$$
d_{X}(x, y)=d_{X}(p \cdot x, p \cdot y) \quad \forall x, y, p \in \mathbb{G} .
$$

Moreover, since it involves only horizontal vector fields, it is homogeneous of degree one with respect to group dilations:

$$
d_{X}\left(\delta_{\lambda}(x), \delta_{\lambda}(y)\right)=\lambda d_{X}(x, y) \quad \forall x, y \in \mathbb{G}, \lambda>0 .
$$

The metric space $\left(\mathbb{G}, d_{X}\right)$ is geodesic: for any couple of points there exists a minimizer in the class of horizontal curves. This is due to Chow Theorem and to the fact that $\mathbb{G}$ is locally compact and complete (see e.g. [17, §9]). 
Definition 2.3 (Horizontal gradient). For a smooth function $f: \mathbb{G} \rightarrow \mathbb{R}$ we define its horizontal gradient as

$$
\nabla_{X} f=\sum_{j=1}^{m_{1}}\left(X_{j} f\right) X_{j}
$$

The map $\nabla_{X} f$ defines a horizontal vector field. The coordinates of $\nabla_{X} f(x)$ in $H_{x} \mathbb{G}$ with respect to the chosen orthonormal basis $\left\{X_{1}(x), \ldots, X_{m_{1}}(x)\right\}$ are given by $\left(\left(X_{1} f\right)(x), \ldots,\left(X_{m_{1}} f\right)(x)\right)$.

Whilst both the sub-Riemannian structure and the horizontal gradient are basis dependent, it is still possible to associate with a function $f$, for which $\left\{X_{j} f(x)\right\}_{j=1}^{m_{1}}$ exist for all $x \in \mathbb{G}$, a well defined linear functional $\mathcal{L}^{f}$ on $H \mathbb{G}$ that does not depend on the basis of the first layer:

$$
\begin{aligned}
\mathcal{L}_{x}^{f}: H_{x} \mathbb{G} & \longrightarrow \mathbb{R} \\
V & \longmapsto\left\langle\nabla_{X} f(x), V\right\rangle_{X} .
\end{aligned}
$$

Definition 2.4 (Homogeneous norms). A homogeneous norm is a continuous function $|\cdot|_{\mathbb{G}}: \mathbb{G} \longrightarrow \mathbb{R}^{+}$with the following properties:

(1) $|x|_{\mathbb{G}}=0 \Leftrightarrow x=0$;

(2) $\left|x^{-1}\right|_{\mathbb{G}}=|x|_{\mathbb{G}} ;$

(3) $\left|\delta_{\lambda}(x)\right|_{\mathbb{G}}=\lambda|x|_{\mathbb{G}}$.

A homogeneous norm induces a left-invariant homogeneous distance by

$$
d(x, y)=\left|y^{-1} \cdot x\right|_{\mathbb{G}}
$$

and a quasi triangular inequality holds (see [7, Proposition 1.6]):

$$
d(x, y) \leq \alpha(d(x, z)+d(z, y)) \text { for some } \alpha>0 .
$$

With $B(x, r)$ we will indicate the metric ball with respect to the homogeneous distance (4): $B(x, r)=\{y \in \mathbb{G}$ such that $d(x, y)<r\}$.

A particular homogeneous norm can be constructed starting from the Carnot-Carathéodory distance, by $|x|_{C C}=d_{X}(x, 0)$, and we recall that any homogeneous norm is equivalent to $|\cdot|_{C C}$, as for the respective distances: if we call $\lambda$ the equivalence parameter, then

$$
\lambda^{-1} d_{X}(x, y) \leq d(x, y) \leq \lambda d_{X}(x, y) .
$$

Definition 2.5 (Homogeneous homomorphisms). Let $\left(\mathbb{G}_{1}, \cdot\right)$ and $\left(\mathbb{G}_{2}, \cdot\right)$ denote Carnot groups with dilations $\delta_{\lambda}^{(i)}, i=1,2$. A map $\varphi: \mathbb{G}_{1} \rightarrow \mathbb{G}_{2}$ is called a homogeneous homomorphism if
i) $\varphi\left(\delta_{\lambda}^{(1)}(x)\right)=\delta_{\lambda}^{(2)}(\varphi(x)) \quad \forall x \in \mathbb{G}_{1}, \lambda>0$;
ii) $\varphi(x \cdot y)=\varphi(x) \cdot \varphi(y) \quad \forall x, y \in \mathbb{G}_{1}$.

Remark 2.6 ( $\mathbb{R}^{M}$-valued homogeneous homomorphisms). If $\left(\mathbb{G}_{2}, \cdot\right)=\left(\mathbb{R}^{M},+\right)$, dilations $\delta_{\lambda}^{(2)}$ are Euclidean. Homogeneous homomorphisms $\varphi:(\mathbb{G}, \cdot) \rightarrow$ $\left(\mathbb{R}^{M},+\right)$ reduce then (see [14, Proposition 2.5]) to functions from the weight 1 variables of $\mathbb{G}$ to $\mathbb{R}^{M}$, with the property

$$
\varphi\left(\delta_{\lambda_{1}}\left(h_{1}\right) \cdot \delta_{\lambda_{2}}\left(h_{2}\right)\right)=\lambda_{1} \varphi\left(h_{1}\right)+\lambda_{2} \varphi\left(h_{2}\right) \quad \forall h_{1}, h_{2} \in \mathbb{G}, \lambda_{1}, \lambda_{2} \in \mathbb{R} .
$$

To emphasize the fact that $\varphi$ acts only on the horizontal components of points $x \in \mathbb{G}$ we will write $\varphi\left(x^{\prime}\right)$. 
Definition 2.7 (G-linear functions). A homogeneous homomorphism $\varphi$ with values in $\mathbb{R}^{M}$ will be called a $\mathbb{G}$-linear function, after [8]. Its action is actually a matrix product in horizontal coordinates, defined by $M m_{1}$ real constants $\left\{A_{i j}^{\varphi}\right\}, i=1 \ldots M, j=1 \ldots m_{1}$ and reads explicitly

$$
\varphi\left(x^{\prime}\right)=\left(\sum_{j=1}^{m_{1}} A_{1 j}^{\varphi} x_{j}, \ldots, \sum_{j=1}^{m_{1}} A_{M j}^{\varphi} x_{j}\right) .
$$

The real-valued matrix $\left(A^{\varphi}\right)_{i j}$ will be called its representative matrix.

In the case of $M=m_{1}$ and $A^{\varphi} \in G L\left(m_{1}, \mathbb{R}\right)$, we obtain a class of maps from $\mathbb{G}$ to $\mathbb{G}$ which we call $\mathbb{G}$-change of basis, by a $\phi^{A}: \mathbb{G} \rightarrow \mathbb{G}$ such that

$$
\begin{aligned}
& \phi^{A}: \quad x \quad \longmapsto \quad \xi \\
& \left(x^{\prime}, x^{\prime \prime}\right) \longmapsto\left(\varphi\left(x^{\prime}\right), x^{\prime \prime}\right) .
\end{aligned}
$$

Such a transformation indeed represents the change of canonical coordinates in $\mathbb{G}$ resulting from a change of basis in the horizontal layer of the algebra. By horizontal rotation we will mean a $\mathbb{G}$-change of basis with $A^{\varphi} \in O\left(m_{1}, \mathbb{R}\right)$.

Remark 2.8. There always exists a homogeneous norm which is invariant under horizontal rotations (see [26, Ch.13 §7.12]). One concrete realization of such a norm is obtained in [14], Theorem 5.1.

We now introduce the commonly used notion of differentiability on Carnot groups, given by Pansu in [24], for the case of real-valued functions.

Definition 2.9 (Pansu differentiability of real valued functions). We say that a function $f: \mathbb{G} \rightarrow \mathbb{R}$ is P-differentiable at $x \in \mathbb{G}$ if there exists a $\mathbb{G}$-linear map $L_{x}^{f}: \mathbb{G} \rightarrow \mathbb{R}$ such that

$$
\lim _{|h|_{\mathbb{G}} \rightarrow 0} \frac{f(x \cdot h)-f(x)-L_{x}^{f}\left(h^{\prime}\right)}{|h|_{\mathbb{G}}}=0 .
$$

Let $H(x)=\sum_{i=1}^{m_{1}} h_{i} X_{i}(x) \in H_{x} \mathbb{G}$ and $h=\exp (H)=\left(h^{\prime}, 0\right) \in \mathbb{G}$. It is well known that if $f$ is $\mathrm{P}$-differentiable at $x$, then

$$
L_{x}^{f}\left(h^{\prime}\right)=\left.\frac{d}{d t} f(x \cdot \exp (t H))\right|_{t=0}=(H f)(x)=\mathcal{L}_{x}^{f}(H(x))
$$

where $\mathcal{L}_{x}^{f}$ is given by (3), so that the Pansu differential does not depend on the basis chosen for $\mathfrak{g}$. In the rest of the paper we will use the notation

$$
L_{x}^{f}\left(h^{\prime}\right)=\left\langle\nabla_{X} f, h^{\prime}\right\rangle_{X} .
$$

Integration on Lie groups is performed with respect to the Haar measure: the following proposition provides its construction on Carnot groups.

Proposition 2.10. Let $d X$ be the Lebesgue measure on the linear space $\mathfrak{g}$. Then the Haar measure $d \mu(x)$ on $\mathbb{G}$ is given by the image through exponential mapping of $d X$. This means that, given $f: \mathbb{G} \rightarrow \mathbb{R}$, the integration on $\mathbb{G}$ can be expressed as an integral in canonical coordinates, that is, on $\mathbb{R}^{N}$ :

$$
\int_{\mathbb{G}} f(x) d \mu(x) \doteq \int_{\mathbb{R}^{N}} f\left(x_{1}, \ldots, x_{N}\right) d x_{1} \ldots d x_{N} \mathbb{1} .
$$

\footnotetext{
${ }^{1}$ For the sake of simplicity we keep the same notation for $f$ on the group and on $\mathbb{R}^{N}$.
} 
The main point here is that integration on $\mathbb{G}$ can be performed as in $\mathbb{R}^{N}$, keeping in mind that group operations affect the measure through canonical coordinates changes: for this reason we will indicate the measure $d \mu(x)$ simply as $d x$. The following corollary states three basic properties of the measure under changes of variables.

Corollary 2.11. Let $\mathbb{G}$ be a Carnot group and $f: \mathbb{G} \longrightarrow \mathbb{R}$ an integrable function on $\mathbb{G}$, then the Haar measure of $\mathbb{G}$

1. is invariant under left and right translations (unimodularity):

$$
\int_{\mathbb{G}} f(\alpha \cdot x) d x=\int_{\mathbb{G}} f(x \cdot \alpha) d x=\int_{\mathbb{G}} f(x) d x \quad \forall \alpha \in \mathbb{G} ;
$$

2. scales under group dilations by the homogeneous dimension of $\mathbb{G}$ :

$$
\int_{\mathbb{G}} f\left(\delta_{\lambda}(x)\right) d x=\lambda^{Q} \int_{\mathbb{G}} f(x) d x \quad \forall \lambda>0 ;
$$

3. is affected by $\mathbb{G}$-changes of basis (7) as the Lebesgue measure on $\left(\mathbb{R}^{N},+\right)$, i.e. putting $\xi=\phi^{A}(x)=\left(A x^{\prime}, x^{\prime \prime}\right)$ we have:

$$
\int_{\mathbb{G}} f(x) d x=\int_{\mathbb{G}} f(\xi)|\operatorname{det} A| d \xi \quad \forall A \in G L\left(m_{1}, \mathbb{R}\right) .
$$

The first two properties provide the basic relation

$$
|B(x, r)|=r^{Q}|B(0,1)| \quad \forall x \in \mathbb{G}
$$

where $|B|$ stands for the measure of the ball $B$, so we will set $c_{B}=|B(0,1)|$.

A notion of smoothness for functions on Carnot groups is that of being $\mathcal{C}^{1}$ with respect to horizontal vector fields. This is stronger than being Pdifferentiable, but requires less regularity than being $\mathcal{C}^{1}$ in Euclidean sense.

Definition 2.12. A continuous function $f: \mathbb{G} \rightarrow \mathbb{R}$ is said to be in $\mathcal{C}_{\mathbb{G}}^{1}(\mathbb{G}, \mathbb{R})$ if $X_{j} f: \mathbb{G} \rightarrow \mathbb{R}$ exist and are continuous for $j=1, \ldots, m_{1}$.

Theorem 2.13. If $f$ is a $\mathcal{C}_{\mathbb{G}}^{1}(\mathbb{G}, \mathbb{R})$ function, then $f$ is P-differentiable.

Remark 2.14. $\mathcal{C}^{1} \subset \mathcal{C}_{\mathbb{G}}^{1}$, and the inclusion is strict.

See [12, §5, Theorem 7] for a proof of Theorem 2.13, and [12, §5, Remark $6]$ for an example of the inclusion in Remark 2.14.

Along with these basic group-related notions of regularity, we recall the commonly used notion of Sobolev space $W^{1, p}$.

Definition 2.15 (Sobolev space $W_{\mathbb{G}}^{1, p}(\mathbb{G})$ ). Let $\mathbb{G}$ be a Carnot group and let $p \geq 1$ be fixed. We denote by $W_{\mathbb{G}}^{1, p}(\mathbb{G})$ the set of functions $f \in L^{p}(\mathbb{G})$ such that $X_{j} f \in L^{p}(\mathbb{G})$ for $j=1, \ldots, m_{1}$, endowed with the norm

$$
\|f\|_{1, p}=\left(\int_{\mathbb{G}}|f(x)|^{p} d x\right)^{1 / p}+\left(\int_{\mathbb{G}}\left|\nabla_{X} f(x)\right|_{X}^{p} d x\right)^{1 / p} .
$$

In particular we recall that the classical density result of smooth functions still holds, see [13].

To conclude the section, we recall a result from [6, Proposition 1.5] (see also [18, §2 Proposition 3], and [7, Proposition 1.13]) which will be useful in the computations. 
Lemma 2.16. Let $\mathbb{G}$ be a Carnot group and let $|\cdot|_{\mathbb{G}}$ be a homogeneous norm. Let $f: \mathbb{G} \rightarrow \mathbb{R}$ be a homogeneous function of degree $-Q$, that is $f\left(\delta_{\lambda}(x)\right)=$ $\lambda^{-Q} f(x) \forall x \in \mathbb{G}$, and locally integrable away from 0 . Then there exists a constant $M(f)$ such that

$$
\int_{\mathbb{G}} f(x) g\left(|x|_{\mathbb{G}}\right) d \mu(x)=M(f) \int_{0}^{\infty} g(r) \frac{d r}{r}
$$

for any $g: \mathbb{R}^{+} \rightarrow \mathbb{R}$ such that each side makes sense.

We compute explicitly the constant $M$ for the function $f(x)=|x|_{\mathbb{G}}^{-Q}$ :

$c_{B}=\int_{B(0,1)} d \mu(x)=\int_{\mathbb{G}} \frac{1}{|x|_{\mathbb{G}}^{Q}}|x|_{\mathbb{G}}^{Q} \chi_{[0,1]}\left(|x|_{\mathbb{G}}\right) d \mu(x)=M\left(\frac{1}{|x|_{\mathbb{G}}^{Q}}\right) \int_{0}^{1} r^{Q-1} d r$ hence $M\left(|x|_{\mathbb{G}}^{-Q}\right)=Q c_{B}$. This allows the explicit computation of integrals on balls of functions depending only on the distance from the center of the ball in terms of integrals on the real line with no need of any coarea formula:

$$
\int_{B\left(x_{0}, R\right)} f\left(\left|x_{0}^{-1} \cdot x\right|_{\mathbb{G}}\right) d \mu(x)=\int_{B(0, R)} f\left(|y|_{\mathbb{G}}\right) d \mu(y)=Q c_{B} \int_{0}^{R} f(r) r^{Q-1} d r .
$$

\section{The Bourgain-Brezis-Mironescu Sobolev space}

We begin providing a notion of radial mollifiers on Carnot groups.

Definition 3.1 (Radial mollifiers). Let $\mathbb{G}$ be a Carnot group and let $|.|_{\mathbb{G}}$ be a given homogeneous norm. A family of functions $\left\{\rho_{n}\right\}_{n>0}, \rho_{n}: \mathbb{G} \longrightarrow \mathbb{R}^{+}$, is said to be a family of radial mollifiers with respect to the norm $|\cdot|_{\mathbb{G}}$ if each $\rho_{n}(x)$ depends only on $|x|_{\mathbb{G}}$ and

$$
\begin{array}{ll}
\text { i) } \quad & \int_{\mathbb{G}} \rho_{n}(x) d x=1 \quad \forall n>0 ; \\
\text { ii) } \quad \int_{\mathbb{G} \backslash B(0, \delta)} \rho_{n}(x) d x \stackrel{n \rightarrow \infty}{\longrightarrow} 0 \quad \forall \delta>0 .
\end{array}
$$

Lemma 3.2. Let $\left\{\tilde{\rho}_{n}\right\}$ be a family of functions $\tilde{\rho}_{n}: \mathbb{R}^{+} \longrightarrow \mathbb{R}^{+}$, and set $\rho_{n}: \mathbb{G} \longrightarrow \mathbb{R}^{+}$as $\rho_{n}(x)=\tilde{\rho}_{n}\left(|x|_{\mathbb{G}}\right)$. Then $\rho_{n}$ are radial mollifiers in the sense of Definition 3.1 if and only if it holds
1. $\int_{0}^{\infty} \tilde{\rho}_{n}(r) r^{Q-1} d r=\frac{1}{Q c_{B}} \quad \forall n>0 ;$
2. $\int_{\delta}^{\infty} \tilde{\rho}_{n}(r) r^{Q-1} d r \stackrel{n \rightarrow \infty}{\longrightarrow} 0 \quad \forall \delta>0$.

Proof. As for 1., by direct computation, using Lemma 2.16, we obtain

$$
\int_{\mathbb{G}} \rho_{n}(x) d x=\int_{\mathbb{G}} \frac{1}{|x|_{\mathbb{G}}^{Q}} \tilde{\rho}_{n}\left(|x|_{\mathbb{G}}\right)|x|_{\mathbb{G}}^{Q} d x=Q c_{B} \int_{0}^{\infty} \tilde{\rho}_{n}(r) r^{Q-1} d r .
$$

Assertion 2. can be handled in the same way.

As customary when dealing with radial functions, with abuse of notation we will denote a radial mollifier $\rho_{n}$ as $\rho_{n}\left(|x|_{\mathbb{G}}\right)$ without distinguish $\rho$ and 
$Q c_{B} \tilde{\rho}$. Moreover, we will make also use of their 1-dimensional counterpart: mollifiers $\rho_{n}^{(1)}: \mathbb{R}^{+} \longrightarrow \mathbb{R}^{+}$satisfying

$$
\int_{0}^{\infty} \rho_{n}^{(1)}(\tau) d \tau=1 \quad \int_{\delta}^{\infty} \rho_{n}^{(1)}(\tau) d \tau \stackrel{n \rightarrow \infty}{\longrightarrow} 0 \quad \forall \delta>0
$$

which, due to the preceding corollary, are in 1-1 correspondence with the radial mollifiers of Definition 3.1 by

$$
\rho_{n}^{(1)}(r)=Q c_{B} \rho_{n}(r) r^{Q-1} .
$$

Before stating the main result of this section, we prove two basic lemmata which will be crucial in the proof. The first lemma is a uniformity result concerning the convergence of the limit (8) in the case of functions with continuous derivatives, and the second lemma is a direct consequence of Lemma 2.16 which provides the exact constant for Theorem 3.5.

Theorem 3.5, that is the counterpart in Carnot groups of [3, Theorem 2], however proved following [4, gives a way to characterize Sobolev spaces and estimate their norms (10) by means of integral approximations: the local structure, expressed by an integral norm of the differential, can be replaced by a finite scale evaluation of function's oscillations, which is shown to be an equivalent condition. This finite scale is governed by the radial mollifiers, and in the limit of its becoming tiny, the differential formulation is recovered.

We stress the fact that the proof of the sharp result of convergence strongly relies on the assumption of rotational invariance for the homogeneous norm (see the proof of Lemma 3.4).

Lemma 3.3. If $f \in \mathcal{C}_{\mathbb{G}}^{1}(\mathbb{G}, \mathbb{R})$ then

$$
\omega_{x}(h) \doteq\left|f(x \cdot h)-f(x)-\left\langle\nabla_{X} f(x), h^{\prime}\right\rangle_{X}\right|
$$

is such that

$$
\begin{aligned}
\text { i) } & \frac{\omega_{x}(h)}{|h|_{\mathbb{G}}} \rightarrow 0 \quad \text { as } h \rightarrow 0, \text { uniformly for } x \text { on compact sets; } \\
\text { ii) } \quad & \frac{\omega_{x}(h)}{|h|_{\mathbb{G}}} \leq C_{K} \quad \forall x \in K \text { compact, } \forall|h|_{\mathbb{G}}<1 .
\end{aligned}
$$

Proof. By Theorem 2.13, $f$ is P-differentiable. Now if $\varphi(t)=f\left(x \cdot \delta_{t}(h)\right)$, then by the Mean Value Theorem there exists a $t^{*} \in(0,1)$ such that

$$
f(x \cdot h)-f(x)=\varphi(1)-\varphi(0)=\left.\frac{d}{d t} \varphi(t)\right|_{t=t^{*}}=\left\langle\nabla_{X} f\left(x \cdot \delta_{t^{*}}(h)\right), h^{\prime}\right\rangle_{X}
$$

where the last transition holds due to the horizontality of the Pansu differential. We then get

$$
\begin{aligned}
\omega_{x}(h) & =\left|\left\langle\left(\nabla_{X} f\left(x \cdot \delta_{t^{*}}(h)\right)-\nabla_{X} f(x)\right), h^{\prime}\right\rangle_{X}\right| \\
& \leq\left|h^{\prime}\right|_{\mathbb{R}^{m_{1}}}\left|\nabla_{X} f\left(x \cdot \delta_{t^{*}}(h)\right)-\nabla_{X} f(x)\right|_{X}
\end{aligned}
$$

which gives

$$
\frac{\omega_{x}(h)}{|h|_{\mathbb{G}}} \leq c\left|\nabla_{X} f\left(x \cdot \delta_{t^{*}}(h)\right)-\nabla_{X} f(x)\right|_{X}
$$

where $c$ is such that $\left|h^{\prime}\right|_{\mathbb{R}^{m_{1}}}<c|h|_{\mathbb{G}}$, see e.g. [6, Lemma 1.3].

The lemma is then proved by the hypothesis of $f$ being a $\mathcal{C}_{\mathbb{G}}^{1}$ function. 
Lemma 3.4. Let $\mathbb{G}$ be a Carnot group, $\left\{\rho_{n}\right\}_{n>0}$ a family of radial mollifiers as in Definition 3.1 and $|\cdot|_{\mathbb{G}}$ a homogeneous norm invariant under horizontal rotations. Call

$$
\kappa_{n}=\int_{B(0,1)} \frac{\left|\left\langle\hat{v}, x^{\prime}\right\rangle\right|^{p}}{|x|_{\mathbb{G}}^{p}} \rho_{n}\left(|x|_{\mathbb{G}}\right) d x
$$

with $\hat{v}$ a unit vector of $\mathbb{R}^{m_{1}}$ and $\langle.,$.$\rangle the Euclidean scalar product on \mathbb{R}^{m_{1}}$. Then

i) for all $n>0, \kappa_{n}$ does not depend on $\hat{v}$. In particular

$$
\kappa_{n}=(p+Q) \int_{B(0,1)}\left|\left\langle\hat{e_{1}}, x^{\prime}\right\rangle\right|^{p} d x \int_{0}^{1} \rho_{n}(r) r^{Q-1} d r
$$

where $\hat{e_{1}}$ stands for the first unit vector of the standard basis of $\mathbb{R}^{m}$;

ii) $\kappa_{n} \rightarrow \kappa \doteq \frac{(p+Q)}{Q c_{B}} \int_{B(0,1)}\left|\left\langle\hat{e_{1}}, x^{\prime}\right\rangle\right|^{p} d x \quad$ as $n \rightarrow \infty$.

Proof. Point $i i$ ) is straightforward from $i$ ) and the definition of radial mollifiers. To prove $i$ ) we make use of Lemma 2.16.

$$
\begin{aligned}
\kappa_{n} & =\int_{\mathbb{G}}\left(\frac{\left|\left\langle\hat{v}, x^{\prime}\right\rangle\right|^{p}}{|x|_{\mathbb{G}}^{p+Q}}\right) \rho_{n}\left(|x|_{\mathbb{G}}\right)|x|_{\mathbb{G}}^{Q} \chi_{[0,1]}\left(|x|_{\mathbb{G}}\right) d x \\
& =M\left(\frac{\left|\left\langle\hat{v}, x^{\prime}\right\rangle\right|^{p}}{|x|_{\mathbb{G}}^{p+Q}}\right) \int_{0}^{1} \rho_{n}(r) r^{Q-1} d r .
\end{aligned}
$$

On the other hand,

$$
\begin{aligned}
\int_{B(0,1)}\left|\left\langle\hat{v}, x^{\prime}\right\rangle\right|^{p} d x & =\int_{\mathbb{G}}\left(\frac{\left|\left\langle\hat{v}, x^{\prime}\right\rangle\right|^{p}}{|x|_{\mathbb{G}}^{p+Q}}\right)|x|_{\mathbb{G}}^{p+Q} \chi_{[0,1]}\left(|x|_{\mathbb{G}}\right) d x \\
& =M\left(\frac{\left|\left\langle\hat{v}, x^{\prime}\right\rangle\right|^{p}}{|x|_{\mathbb{G}}^{p+Q}}\right) \int_{0}^{1} r^{p+q-1} d r=\frac{1}{p+Q} M\left(\frac{\left|\left\langle\hat{v}, x^{\prime}\right\rangle\right|^{p}}{|x|_{\mathbb{G}}^{p+Q}}\right)
\end{aligned}
$$

so that

$$
M\left(\frac{\left|\left\langle\hat{v}, x^{\prime}\right\rangle\right|^{p}}{|x|_{\mathbb{G}}^{p+Q}}\right)=(p+Q) \int_{B(0,1)}\left|\left\langle\hat{v}, x^{\prime}\right\rangle\right|^{p} d x .
$$

This expression does not depend on $\hat{v}$, since by an orthogonal $\mathbb{G}$-change of basis (7) which does not alter the measure (Corollary 2.11) nor the homogeneous norm (so that also the domain of integration does not change), it is possible to choose any other unitary vector of $\mathbb{R}^{m_{1}}$ by rotation: set $A \in O\left(m_{1}, \mathbb{R}\right)$ and call $\hat{w}=A^{T} \hat{v}$, then

$$
\begin{aligned}
\int_{B(0,1)}\left|\left\langle\hat{v}, x^{\prime}\right\rangle\right|^{p} d x & =\int_{B(0,1)}\left|\left\langle\hat{v}, \phi^{A}(x)^{\prime}\right\rangle\right|^{p} d x=\int_{B(0,1)}\left|\left\langle\hat{v}, A x^{\prime}\right\rangle\right|^{p} d x \\
& =\int_{B(0,1)}\left|\left\langle\hat{w}, x^{\prime}\right\rangle\right|^{p} d x .
\end{aligned}
$$


Theorem 3.5. Let $\mathbb{G}$ be a Carnot group, $|\cdot|_{\mathbb{G}}$ a homogeneous norm invariant under horizontal rotations and $\left\{\rho_{n}\right\}_{n>0}$ a family of radial mollifiers as in Definition 3.1. Let then $f \in L^{p}(\mathbb{G})$ be given, with $1<p<\infty$. If in addition

$$
I_{n}=\int_{\mathbb{G}} \int_{\mathbb{G}} \frac{|f(y)-f(x)|^{p}}{\left|x^{-1} \cdot y\right|_{\mathbb{G}}^{p}} \rho_{n}\left(\left|x^{-1} \cdot y\right|_{\mathbb{G}}\right) d x d y \leq C \quad \forall n>n_{0}
$$

then

$$
\begin{aligned}
\text { i) } & f \in W_{\mathbb{G}}^{1, p}(\mathbb{G}) ; \\
\text { ii) } & I_{n} \longrightarrow \kappa \int_{\mathbb{G}}\left|\nabla_{X} f(x)\right|_{X}^{p} d x \text { as } n \rightarrow \infty ;
\end{aligned}
$$

where $\kappa$ is the constant given by Lemma 3.4:

$$
\kappa=\frac{(p+Q)}{Q c_{B}} \int_{B(0,1)}\left|\left\langle\hat{e_{1}}, x^{\prime}\right\rangle\right|^{p} d x .
$$

Proof. If we set $h=x^{-1} \cdot y$ we can write

$$
I_{n}=\int_{\mathbb{G}} \int_{\mathbb{G}} \frac{|f(x \cdot h)-f(x)|^{p}}{|h|_{\mathbb{G}}^{p}} \rho_{n}\left(|h|_{\mathbb{G}}\right) d h d x .
$$

The proof can now be carried out in two steps, producing estimates from above and below. The first step consists in showing that for any $f \in \mathcal{C}_{\mathbb{G}}^{1}(\mathbb{G})$

$$
\kappa \int_{\mathbb{G}}\left|\nabla_{X} f(x)\right|_{X}^{p} d x \leq \liminf _{n \rightarrow \infty} \int_{\mathbb{G}} \int_{\mathbb{G}} \frac{|f(x \cdot h)-f(x)|^{p}}{|h|_{\mathbb{G}}^{p}} \rho_{n}\left(|h|_{\mathbb{G}}\right) d h d x .
$$

Such a result for $\mathcal{C}_{\mathbb{G}}^{1}(\mathbb{G})$ extends then to any $f \in L^{p}(\mathbb{G})$ by density [13. Relation (17) provides one half of $i i$ ) and, together with (16), it implies $i$ ). On the other hand, the second step of the proof will be the complementary estimate: for any $f \in \mathcal{C}_{0_{\mathbb{G}}}^{1}(\mathbb{G})$ it holds

$$
\kappa \int_{\mathbb{G}}\left|\nabla_{X} f(x)\right|_{X}^{p} d x \geq \limsup _{n \rightarrow \infty} \int_{\mathbb{G}} \int_{\mathbb{G}} \frac{|f(x \cdot h)-f(x)|^{p}}{|h|_{\mathbb{G}}^{p}} \rho_{n}\left(|h|_{\mathbb{G}}\right) d h d x .
$$

This together with (17) yields, for any $f \in \mathcal{C}_{0_{\mathbb{G}}}^{1}(\mathbb{G})$,

$$
\lim _{n \rightarrow \infty} \int_{\mathbb{G}} \int_{\mathbb{G}} \frac{|f(x \cdot h)-f(x)|^{p}}{|h|_{\mathbb{G}}^{p}} \rho_{n}\left(|h|_{\mathbb{G}}\right) d h d x=\kappa \int_{\mathbb{G}}\left|\nabla_{X} f(x)\right|_{X}^{p} d x
$$

which extends by density [13] to any $f \in W_{\mathbb{G}}^{1, p}(\mathbb{G})$.

We now prove (17). In order to do that, let us first prove the following preliminary claim: let $K$ be a compact set, $B \equiv B(0,1)$ and $\kappa_{n}$ be the same as in Lemma 3.4, then

$$
\int_{K} d x \int_{B} d h \frac{\left|\left\langle\nabla_{X} f(x), h\right\rangle_{X}\right|^{p}}{|h|_{\mathbb{G}}^{p}} \rho_{n}\left(|h|_{\mathbb{G}}\right)=\kappa_{n} \int_{K} d x\left|\nabla_{X} f(x)\right|_{X}^{p} .
$$

Indeed, since it is sufficient to restrict to compact sets $K$ where $\nabla_{X} f(x) \neq 0$,

$$
\begin{aligned}
\int_{K} d x \int_{B} d h & \frac{\left|\left\langle\nabla_{X} f(x), h^{\prime}\right\rangle_{X}\right|^{p}}{|h|_{\mathbb{G}}^{p}} \rho_{n}\left(|h|_{\mathbb{G}}\right) \\
& =\int_{K} d x\left|\nabla_{X} f(x)\right|_{X}^{p} \int_{B} d h \frac{\left|\left\langle\nu_{f}(x), h^{\prime}\right\rangle_{X}\right|^{p}}{|h|_{\mathbb{G}}^{p}} \rho_{n}\left(|h|_{\mathbb{G}}\right)
\end{aligned}
$$


where $\nu_{f}(x)=\frac{\nabla_{X} f(x)}{\left|\nabla_{X} f(x)\right|_{X}}$ is a unit norm horizontal vector, so that this expression corresponds to that of Lemma 3.4. In particular $\kappa_{n}$ does not depend on $x$, as a result of the rotational invariance shown in the lemma. Claim (19) is is then proved.

Now, using the notation of (14), Lemma 3.3, we get

$$
\left|\left\langle\nabla_{X} f(x), h^{\prime}\right\rangle_{X}\right| \leq|f(x \cdot h)-f(x)|+\omega_{x}(h)
$$

then for any $p>1, \theta>0$ there exists a $C_{p, \theta}>0$ such that

$$
\left|\left\langle\nabla_{X} f(x), h^{\prime}\right\rangle_{X}\right|^{p} \leq(1+\theta)|f(x \cdot h)-f(x)|^{p}+C_{p, \theta} \omega_{x}(h)^{p} .
$$

Combining this relation with (19), with $\theta$ arbitrarily fixed, we get

$$
\begin{aligned}
\kappa_{n} \int_{K} d x\left|\nabla_{X} f(x)\right|_{X}^{p} \leq & (1+\theta) \int_{K} d x \int_{B} d h \frac{|f(x \cdot h)-f(x)|^{p}}{|h|_{\mathbb{G}}^{p}} \rho_{n}\left(|h|_{\mathbb{G}}\right) \\
& +C_{p, \theta} \int_{K} d x \int_{B} d h \frac{\omega_{x}(h)^{p}}{|h|_{\mathbb{G}}^{p}} \rho_{n}\left(|h|_{\mathbb{G}}\right) .
\end{aligned}
$$

Thus (17) will follow after proving that the second term on the right expression vanishes as $n \rightarrow \infty$. To see this, we use both the radial mollifier property of vanishing tails and the behavior of the rest given by Lemma 3.3 .

For any $\delta \in(0,1)$ we can split the integral into two parts:

$$
\int_{K} d x \int_{B} d h \frac{\omega_{x}(h)^{p}}{|h|_{\mathbb{G}}^{p}} \rho_{n}\left(|h|_{\mathbb{G}}\right)=\int_{K} \int_{B(0, \delta)}+\int_{K} \int_{B \backslash B(0, \delta)}=J_{n}^{(1)}+J_{n}^{(2)} .
$$

We can see that $J_{n}^{(1)}$ is arbitrarily small for any $\delta$ sufficiently small, thanks to the uniform convergence of $\omega_{x}(h)$, while for $J_{n}^{(2)}$ we can control $\omega_{x}(h)$ and use the tail property of $\rho_{n}\left(|h|_{\mathbb{G}}\right)$. More precisely, by Lemma 3.3

(1) for all $\eta>0$ there exists $\bar{\delta}(\eta, K)$ such that $\omega_{x}(h)<\left(\frac{\eta}{2|K|}\right)^{1 / p}|h|_{\mathbb{G}}$ for all $|h|_{\mathbb{G}}<\bar{\delta}(\eta, K)$ and all $x \in K$. We then have

$$
J_{n}^{(1)}<\frac{\eta}{2|K|}|K| \int_{B(0, \delta)} \rho_{n}\left(|h|_{\mathbb{G}}\right) d h<\frac{\eta}{2} \quad \forall \delta<\bar{\delta}(\eta, K) \quad \forall n ;
$$

(2) $\omega_{x}(h) \leq C_{K}^{1 / p}|h|_{\mathbb{G}}$ for all $x \in K$. This yields

$$
J_{n}^{(2)} \leq C_{K}|K| \int_{B(0,1) \backslash B(0, \delta)} \rho_{n}\left(|h|_{\mathbb{G}}\right) d h \quad \forall \delta \in(0,1)
$$

and the integral over the annulus can be made arbitrarily small for $n$ large due to the tail property of $\rho_{n}$ (Definition 3.1), so that

$$
J_{n}^{(2)} \leq C_{K}|K| \frac{\eta}{2 C_{K}|K|}=\frac{\eta}{2} \quad \forall n>\bar{n}(\delta, \eta, K) .
$$

Thus, for any fixed $\eta>0$, there exists an $\bar{n}$ depending only on $\eta$ and on the compact set $K$ such that the integral (20) is smaller than $\eta$ provided $n>\bar{n}$ :

$\forall \eta>0 \quad \exists \bar{n}=\bar{n}(\bar{\delta}(\eta, K), \eta, K) \quad$ such that $\quad J_{n}^{(1)}+J_{n}^{(2)}<\eta \quad \forall n>\bar{n}$.

We then end up with

$$
\kappa_{n} \int_{K}\left|\nabla_{X} f(x)\right|_{X}^{p} d x \leq(1+\theta) \int_{K} d x \int_{B} d h \frac{|f(x \cdot h)-f(x)|^{p}}{|h|_{\mathbb{G}}^{p}} \rho_{n}\left(|h|_{\mathbb{G}}\right)+C_{\theta} \eta
$$


which is true for any $n$ sufficiently large. This provides

$$
\int_{K}\left|\nabla_{X} f(x)\right|_{X}^{p} d x \leq(1+\theta) \liminf _{n \rightarrow \infty} \frac{1}{\kappa_{n}} \int_{\mathbb{G}} \int_{\mathbb{G}} \frac{|f(x \cdot h)-f(x)|^{p}}{|h|_{\mathbb{G}}^{p}} \rho_{n}\left(|h|_{\mathbb{G}}\right) d h d x
$$

for any arbitrary $\theta>0$. Moreover, since the right term does not depend on $K$ anymore, we can enlarge the integration domain on the left up to the whole $\mathbb{G}$, so that (17) is proved.

We come now to the second estimate, proving (18) for $f \in \mathcal{C}_{0_{\mathbb{G}}}^{1}(\mathbb{G})$. Again from (14), using the triangular inequality in the other way, we get

$$
|f(x \cdot h)-f(x)| \leq\left|\left\langle\nabla_{X} f(x), h^{\prime}\right\rangle_{X}\right|+\omega_{x}(h)
$$

so that for any $p>1, \theta>0$ there exists a $C_{p, \theta}>0$ such that

$$
|f(x \cdot h)-f(x)|^{p} \leq(1+\theta)\left|\left\langle\nabla_{X} f(x), h^{\prime}\right\rangle_{X}\right|^{p}+C_{\theta} \omega_{x}(h)^{p} .
$$

This means that, by integration on $\mathbb{G}$ and making use of claim (19)

$$
\int_{\mathbb{G}} \int_{\mathbb{G}} \frac{|f(x \cdot h)-f(x)|^{p}}{|h|_{\mathbb{G}}^{p}} \rho_{n}\left(|h|_{\mathbb{G}}\right) d h d x \leq(1+\theta) \kappa_{n} \int_{\mathbb{G}}\left|\nabla_{X} f(x)\right|_{X}^{p} d x+J_{n}
$$

where

$$
J_{n}=C_{\theta} \int_{\mathbb{G}} \int_{\mathbb{G}} \frac{\omega_{x}(h)^{p}}{|h|_{\mathbb{G}}^{p}} \rho_{n}\left(|h|_{\mathbb{G}}\right) d h d x
$$

can be handled as in (20), since integration can be actually restricted to the support of $f$. This means that $J_{n}$ vanishes as $n \rightarrow \infty$, thus proving (18).

Theorem 3.5 actually provides an equivalent notion of the Sobolev space $W_{\mathbb{G}}^{1, p}(\mathbb{G})$ : this can be seen by the following proposition (see [3, Theorem 1]).

Proposition 3.6. Let $f \in W_{\mathbb{G}}^{1, p}(\mathbb{G}), 1 \leq p<\infty$, and $\rho: \mathbb{G} \rightarrow \mathbb{R}$ a nonnegative function such that $\int_{\mathbb{G}} \rho(x) d x=1$. Then

$$
\int_{\mathbb{G}} \int_{\mathbb{G}} d x d y \frac{|f(y)-f(x)|^{p}}{\left|x^{-1} \cdot y\right|_{\mathbb{G}}^{p}} \rho\left(x^{-1} \cdot y\right) \leq \int_{\mathbb{G}}\left|\nabla_{X} f(x)\right|_{X}^{p} d x
$$

Proof. We start with $f \in \mathcal{C}_{\mathbb{G}}^{1}(\mathbb{G})$. Define, for $h \in \mathbb{G}, v(t)=f\left(x \cdot \delta_{t}(h)\right)$ and recall that, by making use of the Pansu differential properties,

$$
\dot{v}(t)=\left\langle\nabla_{X} f\left(x \cdot \delta_{t}(h)\right), h^{\prime}\right\rangle_{X} .
$$

Then

$$
|f(x \cdot h)-f(x)|=\left|\int_{0}^{1}\left\langle\nabla_{X} f\left(x \cdot \delta_{t}(h)\right), h^{\prime}\right\rangle_{X} d t\right| \leq|h|_{\mathbb{G}} \int_{0}^{1}\left|\nabla_{X} f\left(x \cdot \delta_{t}(h)\right)\right|_{X}
$$

so that

$$
\begin{aligned}
\int_{\mathbb{G}}|f(x \cdot h)-f(x)|^{p} d x & \leq|h|_{\mathbb{G}}^{p} \int_{\mathbb{G}} d x \int_{0}^{1}\left|\nabla_{X} f\left(x \cdot \delta_{t}(h)\right)\right|_{X}^{p} d t \\
& =|h|_{\mathbb{G}}^{p} \int_{\mathbb{G}}\left|\nabla_{X} f(x)\right|_{X}^{p} d x .
\end{aligned}
$$

If we now multiply both sides by $\rho(h) /|h|_{\mathbb{G}}^{p}$ and integrate over $\mathbb{G}$ in $d h$ we obtain estimate (21) for any $f \in \mathcal{C}_{\mathbb{G}}^{1}(\mathbb{G})$, so by density for any $f \in$ $W_{\mathbb{G}}^{1, p}(\mathbb{G})$. 


\section{The Poincaré-Ponce inequality}

This section is devoted to extend to Carnot groups a Poincaré-like inequality, introduced in 25], where on the right side stands the finite scale approximation of the $L^{p}$ norm of the gradient described in the last section. The proof is original, in the sense that a different technique with respect to 25] is used, and shows how the finite scale of mollifiers relates to a finite scale on the domain of evaluation. To this end, we need an additional assumption on the mollifiers, namely we assume the $\rho_{n}$ to be nonincreasing functions. This technical point was already discussed in [3] and [25].

We start with a preliminary one dimensional lemma.

Lemma 4.1 (One dimensional inequality). Let $f \in L_{\text {loc }}^{p}(\mathbb{R})$ be given, $1 \leq$ $p<\infty$, and let $\varphi: \mathbb{R}^{+} \rightarrow \mathbb{R}^{+}$be a nonincreasing function in $L_{\text {loc }}^{1}\left(\mathbb{R}^{+}\right)$. Then, setting $\bar{f}=\int_{-\frac{1}{2}}^{\frac{1}{2}} f(t) d t$, it holds

$$
\int_{-\frac{1}{2}}^{\frac{1}{2}}|f(t)-\bar{f}|^{p} d t \leq \frac{2}{\int_{0}^{1} d \tau \rho(\tau)} \int_{-\frac{1}{2}}^{\frac{1}{2}} d t \int_{-\frac{1}{2}}^{\frac{1}{2}} d s \frac{|f(t)-f(s)|^{p}}{|t-s|^{p}} \varphi(|t-s|) .
$$

Proof. To prove this lemma we will make use of [3, Lemma 2]. We first claim that if we set

$$
g(\tau)=\frac{1}{\tau^{p}} \int_{-\frac{1}{2}}^{\frac{1}{2}-\tau} d t|f(t+\tau)-f(t)|^{p}
$$

then for all $\tau \in(0,1)$ it holds $g(\tau) \leq g(\tau / 2)$. Indeed if we take $0<\sigma<1 / 2$

$$
\begin{aligned}
& g(2 \sigma)=\frac{1}{(2 \sigma)^{p}} \int_{-\frac{1}{2}}^{\frac{1}{2}-2 \sigma} d t|f(t+2 \sigma)-f(t)|^{p} \\
& \leq \frac{1}{2 \sigma^{p}}\left\{\int_{-\frac{1}{2}}^{\frac{1}{2}-2 \sigma} d t|f(t+2 \sigma)-f(t+\sigma)|^{p}+\int_{-\frac{1}{2}}^{\frac{1}{2}-2 \sigma} d t|f(t+\sigma)-f(t)|^{p}\right\} \\
& \leq \frac{1}{\sigma^{p}} \int_{-\frac{1}{2}}^{\frac{1}{2}-\sigma} d t|f(t+\sigma)-f(t)|^{p}=g(\sigma) .
\end{aligned}
$$

The previous claim allows to apply Lemma 2 of [3]:

$$
\int_{0}^{1} d \tau g(\tau) \varphi(\tau) \geq \frac{1}{2} \int_{0}^{1} d \tau g(\tau) \int_{0}^{1} d \tau \varphi(\tau)
$$

The proof of inequality (22) now follows:

$$
\begin{aligned}
\int_{-\frac{1}{2}}^{\frac{1}{2}} d t|f(t)-\bar{f}|^{p} \leq \int_{-\frac{1}{2}}^{\frac{1}{2}} d t \int_{-\frac{1}{2}}^{\frac{1}{2}} d s|f(t)-f(s)|^{p} \\
\quad \leq 2 \int_{0}^{1} d \tau \frac{1}{\tau^{p}} \int_{-\frac{1}{2}}^{\frac{1}{2}-\tau} d t|f(t+\tau)-f(t)|^{p} \\
\quad=2 \int_{0}^{1} d \tau g(\tau) \leq \frac{4}{\int_{0}^{1} d \tau \rho(\tau)} \int_{0}^{1} d \tau g(\tau) \rho(\tau) \\
=\frac{2}{\int_{0}^{1} d \tau \rho(\tau)} \int_{-\frac{1}{2}}^{\frac{1}{2}} d t \int_{-\frac{1}{2}}^{\frac{1}{2}} d s \frac{|f(t)-f(s)|^{p}}{|t-s|^{p}} \rho(|t-s|)
\end{aligned}
$$


Corollary 4.2. Let $\left\{\rho_{n}^{(1)}\right\}$ be a family of 1-dimensional mollifiers as in (12) such that each $\rho_{n}^{(1)}$ is a nonincreasing function. Then for any $t_{0} \in \mathbb{R}, T>0$ and for any $C>2$ there exists an $n_{0}$ such that

$$
\int_{I}\left|f(t)-\bar{f}_{I}\right|^{p} d t \leq C T^{p} \int_{I} d t \int_{I} d s \frac{|f(t)-f(s)|^{p}}{|t-s|^{p}} \rho_{n}^{(1)}(|t-s|)
$$

for any $n>n_{0}$ and for any $f \in L_{l o c}^{p}(\mathbb{R}), 1 \leq p<\infty$, where $I$ is the interval $I\left(t_{0}, T\right)=\left[t_{0}-T / 2, t_{0}+T / 2\right]$ and we have set $\bar{f}_{I}=f_{I} f(t) d t$.

In particular, $n_{0}=n_{0}(C, T)$ is determined by the condition

$$
\int_{0}^{T} \rho_{n}^{(1)}(\tau) d \tau>\frac{2}{C} \quad \forall n>n_{0}
$$

and always exists by definition of mollifiers.

This corollary says that Lemma 4.1 is a Poincaré-like inequality on intervals of $\mathbb{R}$. The proof essentially relies on applying scaling and translations.

Proof. If we take any $\varphi: \mathbb{R}^{+} \rightarrow \mathbb{R}^{+}$in $L_{\text {loc }}^{1}\left(\mathbb{R}^{+}\right)$, nonincreasing, and set

$$
\tilde{f}(t)=f\left(T t+t_{0}\right), \quad \tilde{\varphi}(t)=\varphi(T t) .
$$

we obtain auxiliary functions which still satisfy the hypotheses of Lemma 4.1, so that (22) holds. Hence we obtain, for the couple $(f, \varphi)$, a Poincaré-like inequality on the interval :

$$
\int_{I}\left|f(t)-\bar{f}_{I}\right|^{p} d t \leq \frac{2}{\int_{0}^{T} \varphi(\tau) d \tau} T^{p} \int_{I} d t \int_{I} d s \frac{|f(t)-f(s)|^{p}}{|t-s|^{p}} \varphi(|t-s|) .
$$

Now it suffices to write inequality (25) for a family of one dimensional mollifiers $\rho_{n}^{(1)}$ as in (12).

Condition (24), that allows to determine the threshold $n_{0}$, means that the smaller is the interval of evaluation, the smaller must be the analyzing scale powered by the mollifiers. This is made clearer by the following remark.

Remark 4.3. Set $\varphi$ within the hypotheses of Lemma 4.1, then for any $\sigma>0$

$$
\int_{-\frac{1}{2}}^{\frac{1}{2}} d t|f(t)-\bar{f}|^{p} \leq \frac{2}{\int_{0}^{\sigma} \varphi(\tau) d \tau} \sigma \int_{-\frac{1}{2}}^{\frac{1}{2}} d t \int_{-\frac{1}{2}}^{\frac{1}{2}} d s \frac{|f(t)-f(s)|^{p}}{|t-s|^{p}} \varphi(\sigma|t-s|) .
$$

This follows immediately from inequality (22) with $\tilde{\varphi}(\tau)=\varphi(\sigma \tau)$.

If we take here $\varphi$ as a mollifier $\rho_{n}^{(1)}$, we obtain a right term with $\sigma \rho_{n}^{(1)}(\sigma$. ), which has the same mass of $\rho$ but a different analyzing scale (larger as $\sigma$ tightens). For small $\sigma$, this can be compensated by taking higher values of $n$, which indeed provide a constant that does not depend on $\sigma$. The point we want to stress is that to get Poincaré-like estimates with a nonlocal term on the right, the scale of nonlocality (indexed by $\mathrm{n}$ ) is necessarily influenced by the scale of the problem.

In this section we will also need a result on the structure of Carnot-Carathéodory balls from [22, §3], which we state here in a shortened form for the case of interest. 
Theorem 4.4. Let $\mathbb{G}$ be a Carnot group of dimension $N$ and step $k$, denote by $m_{j}$ the dimension of the $j$-th layer and by $\left\{X_{1}, \ldots, X_{m_{1}}\right\}$ a basis of the horizontal layer. Let $d_{X}$ be the $C C$ distance (1) and $B_{X}$ the related balls. Then there exist three multi-indexes of length $M=\sum_{l=1}^{k}\left(3 \cdot 2^{l-1}-2\right) m_{l}$

$$
\begin{aligned}
I=\left(i_{1}, \ldots, i_{M}\right), & & i_{n} \in\left\{1, \ldots, m_{1}\right\} \\
J=\left(j_{1}, \ldots, j_{M}\right), & & j_{n} \in\{1, \ldots, N\} \\
\omega=\left(\omega_{1}, \ldots, \omega_{M}\right), & & \omega_{n} \in\{0,1\}
\end{aligned}
$$

and two geometric constants $0<b<a<1$ such that if we set

$$
\begin{aligned}
& E_{I, J, \omega}: \quad \mathbb{R}^{N} \longrightarrow \mathbb{G} \\
& \left(t_{1}, \ldots, t_{N}\right) \longmapsto \exp \left((-1)^{\omega_{1}} t_{j_{1}} X_{i_{1}}\right) \cdot \ldots \cdot \exp \left((-1)^{\omega_{M}} t_{j_{M}} X_{i_{M}}\right)
\end{aligned}
$$

then

$$
B_{X}(0, b R) \subset E_{I, J, \omega}(Q(0, a R)) \subset B_{X}(0, R)
$$

for all $R>0$, where

$$
Q(0, \delta)=\left\{\left(t_{1}, \ldots, t_{N}\right) \in \mathbb{R}^{N}: \max \left|t_{j}\right|<\delta\right\} .
$$

The main geometric meaning of this theorem is contained in the structure of the multi-indexes $I, J, \omega$, which we have skipped here since it goes beyond our needs. The idea is that each point of a Carnot group can be reached by exponential curves of horizontal vector fields, and when a commutator of two vector fields is needed, it can be approximated by a finite length "square path" along the two fields, taken successively with opposite signs. This is the main difference between this result and the classical result due to Nagel, Stein and Wainger ([23, Theorem 7]), where instead the authors work directly with integral curves of commutators. The key relation (26) indicates that the approximated exponential coordinates $\left(t_{1}, \ldots, t_{N}\right)$ are uniformly controlled by the size of the CC-ball that they are able to span, and this will be used as a control on the time-lengths of connecting curves.

We are now ready to prove the main theorem of this section.

Theorem 4.5. Let $\mathbb{G}$ be a Carnot group and $|\cdot|_{\mathbb{G}}$ a homogeneous norm with parameter $\lambda$ as in (6). Then there exist two positive geometric constants $\beta, \mu$ and a positive constant $C_{p, Q}$ depending only on $p, Q$ and the geometric constants $a, b, M$ given by Theorem 4.4 such that

$$
\int_{B} d x\left|f(x)-f_{B}\right|^{p} \leq C_{p, Q} R^{p} \int_{\mu B} \int_{\mu B} d x d y \frac{|f(y)-f(x)|^{p}}{\left|y^{-1} \cdot x\right|_{\mathbb{G}}^{p+Q-1}} \frac{\varphi\left(\left|y^{-1} \cdot x\right|_{\mathbb{G}}\right)}{\int_{0}^{\beta R} \varphi(\tau) d \tau}
$$

for all balls $B$ of radius $R$, all $f$ in $L_{l o c}^{p}(\mathbb{G}), 1 \leq p<\infty$ and all nonincreasing $\varphi: \mathbb{R}^{+} \rightarrow \mathbb{R}^{+}$in $L_{\text {loc }}^{1}\left(\mathbb{R}^{+}\right)$.

Proof. The proof is largely inspired by a simple proof of the classical Poincaré inequality, due to Varopoulos (see [29], and also [16], 8] ). The constant $C$ that will appear is intended as a positive constant that can change from line to line. To start, by Hölder inequality

$$
I=\int_{B\left(x_{0}, R\right)} d x\left|f(x)-f_{B}\right|^{p} \leq \frac{1}{c_{B} R^{Q}} \int_{B\left(x_{0}, R\right)} d x \int_{B\left(x_{0}, R\right)} d y|f(x)-f(y)|^{p} .
$$


Then, passing to Carnot-Carathéodory balls and setting $c_{B_{X}}=\left|B_{X}(0,1)\right|$

$$
\begin{aligned}
I & \leq \frac{1}{c_{B} R^{Q}} \int_{B_{X}\left(x_{0}, \lambda R\right)} d x \int_{B_{X}\left(x_{0}, \lambda R\right)} d y|f(x)-f(y)|^{p} \\
& =\lambda^{Q} \frac{c_{B_{X}}}{c_{B}} f_{B_{X}\left(x_{0}, \lambda R\right)} d y \int_{y^{-1} \cdot B_{X}\left(x_{0}, \lambda R\right)} d z|f(y \cdot z)-f(y)|^{p} \\
& \leq \lambda^{Q} \frac{c_{B_{X}}}{c_{B}} f_{B_{X}\left(x_{0}, \lambda R\right)} d y \int_{B_{X}(0,2 \lambda R)} d z|f(y \cdot z)-f(y)|^{p}
\end{aligned}
$$

where the last transition holds because $y^{-1} \cdot B_{X}\left(x_{0}, \lambda R\right) \subset B_{X}(0,2 \lambda R)$ : indeed, by left-invariance of the CC-distance

$$
d_{X}(z, 0)=d_{X}(y \cdot z, y) \leq d_{X}\left(y \cdot z, x_{0}\right)+d_{X}\left(x_{0}, y\right)<2 \lambda R
$$

so we end up with

$$
I \leq C f_{B_{X}(0,2 \lambda R)} d z \int_{B_{X}\left(x_{0}, \lambda R\right)} d y|f(y \cdot z)-f(y)|^{p} .
$$

By Theorem 4.4 we can reach any $z \in B_{X}(0,2 \lambda R)$ with a finite composition of integral curves of horizontal vector fields, within a finite time. More precisely we can write

$$
z=\prod_{k=1}^{M} \exp \left((-1)^{\omega_{k}} t_{j_{k}} X_{i_{k}}\right)
$$

where, by (26) , if we set $T=\frac{2 \lambda}{b}$ we have that the times needed to cover this path are uniformly bounded by

$$
\left|t_{n}\right|<a T R \quad \forall n \in\{1, \ldots, N\} .
$$

Making use of partial compositions $\zeta_{n}=\prod_{k=1}^{n} \exp \left((-1)^{\omega_{k}} t_{j_{k}} X_{i_{k}}\right)$, we obtain

$$
\begin{aligned}
& |f(y \cdot z)-f(y)|^{p}=\left|f\left(y \cdot \prod_{k=1}^{M} \exp \left((-1)^{\omega_{k}} t_{j_{k}} X_{i_{k}}\right)\right)-f(y)\right|^{p} \\
& =\left|\sum_{n=1}^{M-1}\left[f\left(y \cdot \zeta_{n+1}\right)-f\left(y \cdot \zeta_{n}\right)\right]+f\left(y \cdot \exp \left((-1)^{\omega_{1}} t_{j_{1}} X_{i_{1}}\right)\right)-f(y)\right|^{p} \\
& \leq M^{p-1}\left[\sum_{n=1}^{M-1}\left|f\left(y \cdot \zeta_{n+1}\right)-f\left(y \cdot \zeta_{n}\right)\right|^{p}\right. \\
& \left.\quad+\left|f\left(y \cdot \exp \left((-1)^{\omega_{1}} t_{j_{1}} X_{i_{1}}\right)\right)-f(y)\right|^{p}\right]
\end{aligned}
$$

so that we can separate the estimate of the mean oscillations into $M$ pieces

$$
I \leq C \sum_{n=0}^{M-1} I_{n}
$$


where

$$
\left\{\begin{aligned}
I_{n}= & f_{B_{X}(0,2 \lambda R)} d z \int_{B_{X}\left(x_{0}, \lambda R\right)} d y \mid f\left(y \cdot \prod_{k=1}^{n+1} \exp \left((-1)^{\omega_{k}} t_{j_{k}} X_{i_{k}}\right)\right) \\
& -\left.f\left(y \cdot \prod_{k=1}^{n} \exp \left((-1)^{\omega_{k}} t_{j_{k}} X_{i_{k}}\right)\right)\right|^{p} \text { if } n>0 \\
I_{0}= & f_{B_{X}(0,2 \lambda R)} d z \int_{B_{X}\left(x_{0}, \lambda R\right)} d y\left|f\left(y \cdot \exp \left((-1)^{\omega_{1}} t_{j_{1}} X_{i_{1}}\right)\right)-f(y)\right|^{p} .
\end{aligned}\right.
$$

We note here that terms $I_{n}$ with $n>0$ can be reduced to the form of term $I_{0}$ by means of the change of variables $\eta=y \cdot \zeta_{n}$

$$
\begin{aligned}
I_{n} & =f_{B_{X}(0,2 \lambda R)} d z \int_{B_{X}\left(x_{0}, \lambda R\right) \cdot \zeta_{n}} d \eta\left|f\left(\eta \cdot \exp \left((-1)^{\omega_{n+1}} t_{j_{n+1}} X_{i_{n+1}}\right)\right)-f(\eta)\right|^{p} \\
& \leq f_{B_{X}(0,2 \lambda R)} d z \int_{B_{X}\left(x_{0},(T+\lambda) R\right)} d \eta\left|f\left(\eta \cdot \exp \left((-1)^{\omega_{n+1}} t_{j_{n+1}} X_{i_{n+1}}\right)\right)-f(\eta)\right|^{p}
\end{aligned}
$$

where the last transition holds since, by (28) and making use of (26)

$$
d_{X}\left(\eta, x_{0}\right) \leq d_{X}(\eta, y)+d_{X}\left(y, x_{0}\right)<d_{X}\left(\zeta_{n}, 0\right)+\lambda R<T R+\lambda R .
$$

Each $I_{n}$, for $n=0, \ldots, M-1$, is then bounded by an integral of type

$$
J=f_{B_{X}(0,2 \lambda R)} d z \int_{B_{X}\left(x_{0},(T+\lambda) R\right)} d \eta|f(\eta \cdot \exp (\tau X))-f(\eta)|^{p}
$$

where $|\tau|<a T R$ given by (28) contains the dependence on $z$.

Our purpose is to reduce this expression to a form suitable to use Lemma (4.1). To do this, we first claim that

$$
J \leq 2^{p+2} \int_{B_{X}\left(x_{0},(3 T+\lambda) R\right)} d x \int_{-\frac{1}{2}}^{\frac{1}{2}} d t \int_{-\frac{1}{2}}^{\frac{1}{2}} d s|f(x \cdot \gamma(t))-f(x \cdot \gamma(s))|^{p}
$$

where we have set $\gamma(t)=\exp (4 a T R t X)$. We observe that this claim does not contain anymore integration on $z$, so it will provide an estimate where the multi-indexes $j_{n}$ and $\omega_{n}$ do not appear. To prove it, we begin with

$$
\begin{aligned}
\mid & f(\eta \cdot \exp (\tau X))-\left.f(\eta)\right|^{p} \\
= & \frac{1}{(2 a T R)^{2}} \int_{-a T R}^{a T R} d t \int_{-a T R}^{a T R} d \sigma|f(\eta \cdot \exp (\tau X))-f(\eta)|^{p} \\
\leq & \frac{2^{p-1}}{(2 a T R)^{2}}\left[\int_{-a T R}^{a T R} d t \int_{-a T R}^{a T R} d \sigma|f(\eta \cdot \exp (\tau X))-f(\eta \cdot \exp ((\tau+\sigma) X))|^{p}\right. \\
& \left.\quad+\int_{-a T R}^{a T R} d t \int_{-a T R}^{a T R} d \sigma|f(\eta \cdot \exp ((\tau+\sigma) X))-f(\eta)|^{p}\right] \\
= & \frac{2^{p-1}}{(2 a T R)^{2}}[A(\eta, \tau)+B(\eta, \tau)] .
\end{aligned}
$$


We now look separately at these two terms: for the first one we have, indicating $\omega=a T R$ and $B_{X}=B_{X}\left(x_{0}, R\right)$

$$
\begin{aligned}
J_{A}(\tau)= & \int_{B_{X}\left(x_{0},(T+\lambda) R\right)} d \eta A(\eta, \tau) \\
= & \int_{-\omega}^{\omega} d t \int_{-\omega}^{\omega} d \sigma \int_{(T+\lambda) B_{X}} d \eta|f(\eta \cdot \exp (\tau X))-f(\eta \cdot \exp ((\tau+\sigma) X))|^{p} \\
= & \int_{-\omega}^{\omega} d t \int_{-\omega}^{\omega} d \sigma \int_{(T+\lambda) B_{X}} d \eta \mid f(\eta \cdot \exp ((\tau-t) X) \cdot \exp (t X)) \\
& \quad-\left.f(\eta \cdot \exp ((\tau-t) X) \cdot \exp ((t+\sigma) X))\right|^{p} \\
\leq & \int_{-\omega}^{\omega} d t \int_{-\omega}^{\omega} d \sigma \int_{(3 T+\lambda) B_{X}} d x|f(x \cdot \exp (t X))-f(x \cdot \exp ((t+\sigma) X))|^{p}
\end{aligned}
$$

where the last transiton holds since, after performing the change of variables $x=\eta \cdot \exp ((\tau-t) X)$, we have

$$
d_{X}\left(x, x_{0}\right) \leq d_{X}(x, \eta)+d_{X}\left(\eta, x_{0}\right)<d_{X}(\exp ((\tau-t) X), 0)+(T+\lambda) R
$$

and $|\tau-t| \leq 2 a T R$ so, again by (26),$d_{X}(\exp ((\tau-t) X), 0) \leq 2 T R$.

Moreover, if we set $s=t+\sigma$, we end up with

$$
J_{A} \leq \int_{-2 \omega}^{2 \omega} d t \int_{-2 \omega}^{2 \omega} d s \int_{(3 T+\lambda) B_{X}} d x|f(x \cdot \exp (t X))-f(x \cdot \exp (s X))|^{p}
$$

where, as desired, we have no more dependence on $\tau$, hence on $z$.

For the second term we proceed in a similar way:

$$
\begin{aligned}
J_{B}(\tau) & =\int_{B_{X}\left(x_{0},(T+\lambda) R\right)} d \eta B(\eta, \tau) \\
& =\int_{-\omega}^{\omega} d t \int_{-\omega}^{\omega} d \sigma \int_{(T+\lambda) B_{X}} d \eta \mid f\left(\eta \cdot \exp ((\sigma+\tau) X)-\left.f(\eta)\right|^{p}\right. \\
& \leq \int_{-\omega}^{\omega} d t \int_{-\omega}^{\omega} d \sigma \int_{(3 T+\lambda) B_{X}} d x \mid f\left(x \cdot \exp ((t+\tau) X)-f\left(\left.x \cdot \exp ((t-\sigma) X)\right|^{p}\right.\right.
\end{aligned}
$$

where in the last transition we have made the change $x=\eta \cdot \exp ((\sigma-t) X)$ so that, with a translation in $\sigma$ and $t$, we get

$$
J_{B} \leq \int_{-2 \omega}^{2 \omega} d t \int_{-2 \omega}^{2 \omega} d s \int_{(3 T+\lambda) B_{X}} d x|f(x \cdot \exp (t X))-f(x \cdot \exp (s X))|^{p} .
$$

Since

$$
J \leq \frac{2^{p-1}}{(2 a T R)^{2}} f_{B_{X}(0,2 \lambda R)} d z\left(J_{A}+J_{B}\right)
$$

combining (31) and (32) claim (30) is proved.

We can now make use of the one dimensional estimate (22), in the form given by Remark 4.3, indeed claim (30) allows to reproduce the proof of Lemma 4.1, starting from (23). Hence

$$
J \leq \frac{2^{p+3}}{\int_{0}^{\sigma} \varphi(\tau) d \tau} \sigma \int_{B} d x \int_{-\frac{1}{2}}^{\frac{1}{2}} d t \int_{-\frac{1}{2}}^{\frac{1}{2}} d s \frac{|f(x \cdot \gamma(t))-f(x \cdot \gamma(s))|^{p}}{|t-s|^{p}} \varphi(\sigma|t-s|)
$$

with $B=B_{X}\left(x_{0},(3 T+\lambda) R\right)$, for any nonincreasing $\varphi: \mathbb{R}^{+} \rightarrow \mathbb{R}^{+}$in $L_{l o c}^{1}$. 
We remark that it is possible to apply Lemma 4.1 since the request of $f$ to be in $L_{l o c}^{p}(\mathbb{G})$ guarantees that the function

$$
t \mapsto f(x \cdot \gamma(t))
$$

is in $L_{l o c}^{p}\left(\mathbb{R}^{+}\right)$for almost every $x$. This is a consequence of Fubini theorem: indeed let $B \subset \mathbb{G}$ be a ball and $I \subset \mathbb{R}^{+}$be an interval of the real line, then

$$
\begin{aligned}
\int_{B} d x \int_{I} d t|f(x \cdot \gamma(t))|^{p} & =\int_{I} d t \int_{B} d x|f(x \cdot \gamma(t))|^{p}=\int_{I} d t \int_{B \cdot \gamma(t)} d y|f(y)|^{p} \\
& \leq \int_{I} d t \int_{B^{\prime}} d y|f(y)|^{p} \leq|I| \int_{B^{\prime}} d y|f(y)|^{p}<\infty
\end{aligned}
$$

where $B^{\prime}$ is a ball containing $B \cdot \gamma(t)$ for all $t \in I$.

At this point we still leave the scale $\sigma$ undetermined, since it will become clear later the exact amount we will need. Inequality (29) reduces then to

$$
I \leq C \frac{\sigma}{\int_{0}^{\sigma} \varphi(\tau) d \tau} \int_{-\frac{1}{2}}^{\frac{1}{2}} d t \int_{-\frac{1}{2}}^{\frac{1}{2}} d s \frac{\varphi(\sigma|t-s|)}{|t-s|^{p}} \sum_{n=1}^{M} J_{n}(t, s)
$$

with

where we have denoted

$$
J_{n}(t, s)=\int_{B} d x\left|f\left(x \cdot \gamma_{n}(t)\right)-f\left(x \cdot \gamma_{n}(s)\right)\right|^{p}
$$

$$
\gamma_{n}(t)=\exp \left(4 a T R t X_{i_{n}}\right) .
$$

Let us now define, for arbitrary $\epsilon$, the sets

$$
\Omega_{n}=B_{X}\left(\gamma_{n}\left(\frac{t+s}{2}\right), \epsilon|t-s| R\right) .
$$

These $\Omega_{n}$ are balls centered in between $\gamma_{n}(t)$ and $\gamma_{n}(s)$, with radius comparable to their distance, and such that $\Omega_{n}=\Omega_{n}(t, s)=\Omega_{n}(s, t)$. We then see that

$$
\begin{aligned}
& \left|f\left(x \cdot \gamma_{n}(t)\right)-f\left(x \cdot \gamma_{n}(s)\right)\right|^{p}=f_{\Omega_{n}} d \xi\left|f\left(x \cdot \gamma_{n}(t)\right)-f\left(x \cdot \gamma_{n}(s)\right)\right|^{p} \\
& \leq 2^{p-1}\left(f_{\Omega_{n}} d \xi\left|f\left(x \cdot \gamma_{n}(t)\right)-f(x \cdot \xi)\right|^{p}+f_{\Omega_{n}} d \xi\left|f\left(x \cdot \gamma_{n}(s)\right)-f(x \cdot \xi)\right|^{p}\right) .
\end{aligned}
$$

Due to the symmetry by exchange of $t$ with $s$, we then obtain

$$
J_{n}(t, s) \leq 2^{p} \int_{B} d x f_{\Omega_{n}} d \xi\left|f\left(x \cdot \gamma_{n}(t)\right)-f(x \cdot \xi)\right|^{p} .
$$

We can now perform two changes of variables. The first one is $y=x \cdot \gamma_{n}(t)$, so that

$$
\begin{aligned}
J_{n}(t, s) & \leq 2^{p} f_{\Omega_{n}} d \xi \int_{B \cdot \gamma_{n}(t)} d y\left|f(y)-f\left(y \cdot \gamma_{n}(t)^{-1} \cdot \xi\right)\right|^{p} \\
& \leq 2^{p} f_{\Omega_{n}} d \xi \int_{B_{X}\left(x_{0},(5 T+\lambda) R\right)} d y\left|f(y)-f\left(y \cdot \gamma_{n}(t)^{-1} \cdot \xi\right)\right|^{p}
\end{aligned}
$$

where the last transition is due to

$$
d_{X}\left(y, x_{0}\right) \leq d_{X}(y, x)+d_{X}\left(x, x_{0}\right)<d_{X}\left(\gamma_{n}(t), 0\right)+(3 T+\lambda) R
$$

and, using (26) , since $|4 a T R t|<2 a T R$ then $d_{X}\left(\gamma_{n}(t), 0\right)<2 T R$. 
The second change is $h=\gamma_{n}(t)^{-1} \cdot \xi$, so that

$$
\begin{aligned}
J_{n}(t, s) & \leq 2^{p} \int_{B_{X}\left(x_{0},(5 T+\lambda) R\right)} d y f_{\gamma_{n}(t)^{-1} \cdot \Omega_{n}} d h|f(y \cdot h)-f(y)|^{p} \\
& \leq 2^{p} \int_{B_{X}\left(x_{0},(5 T+\lambda) R\right)} d y \int_{B_{X}(0,(\epsilon+2 a T)|t-s| R)} d h \frac{|f(y \cdot h)-f(y)|^{p}}{c_{B_{X}}(\epsilon|t-s| R)^{Q}}
\end{aligned}
$$

where the last transition holds because

$$
\begin{aligned}
d_{X}(h, 0) & =d_{X}\left(\gamma_{n}(t)^{-1} \cdot \xi, 0\right)=d_{X}\left(\xi, \gamma_{n}(t)\right) \\
& \leq d_{X}\left(\xi, \gamma_{n}\left(\frac{t+s}{2}\right)\right)+d_{X}\left(\gamma_{n}\left(\frac{t+s}{2}\right), \gamma_{n}(t)\right) \\
& <\epsilon|t-s| R+d_{X}\left(\gamma_{n}\left(\frac{|t-s|}{2}\right), 0\right)
\end{aligned}
$$

and

$$
\begin{aligned}
d_{X}\left(\gamma_{n}\left(\frac{|t-s|}{2}\right), 0\right) & \leq \int_{0}^{\frac{|t-s|}{2}}\left|\dot{\gamma}_{n}(\tau)\right|_{X} d \tau \\
& =4 a T R \int_{0}^{\frac{|t-s|}{2}}\left|X_{i_{n}}\left(\gamma_{n}(\tau)\right)\right|_{X} d \tau=2 a T|t-s| R
\end{aligned}
$$

We have then obtained for $J_{n}$ a uniform estimate in $n$, so (33) becomes

$$
\begin{aligned}
I \leq & C \frac{\sigma}{R^{Q} \int_{0}^{\sigma} \varphi(\tau) d \tau} \int_{-\frac{1}{2}}^{\frac{1}{2}} d t \int_{-\frac{1}{2}}^{\frac{1}{2}} d s \frac{\varphi(\sigma|t-s|)}{|t-s|^{p+Q}} d y|f(y \cdot h)-f(y)|^{p} \\
& \int_{B_{X}\left(x_{0},(5 T+\lambda) R\right)} d y \int_{B_{X}(0,(\epsilon+2 a T)|t-s| R)} d h|c| c \int_{0}^{1} d \tau \frac{\varphi(\sigma \tau)}{\tau^{p+Q}} \\
\leq & C \frac{\sigma}{R^{Q} \int_{0}^{\sigma} \varphi(\tau) d \tau} \int_{B_{X}\left(x_{0},(5 T+\lambda) R\right)} d h|f(y \cdot h)-f(y)|^{p} \\
& \int_{B_{X}(0,(\epsilon+2 a T) \tau R)}
\end{aligned}
$$

We apply now Fubini theorem to the last two integrations:

$$
\int_{0}^{1} d \tau \int_{B_{X}(0,(\epsilon+2 a T) \tau R)} d h=\int_{B_{X}(0,(\epsilon+2 a T) R)} d h \int_{\frac{d_{X}(h, 0)}{(\epsilon+2 a T) R}}^{1} d \tau
$$

so that in the end we get, setting $\nu=\max \{5 T+\lambda, \epsilon+2 a T\}$

$$
I \leq \frac{C}{\int_{0}^{\sigma} \varphi(\tau) d \tau} \int_{B_{X}\left(x_{0}, \nu R\right)} d y \int_{B_{X}(0, \nu R)} d h|f(y \cdot h)-f(y)|^{p} \Phi_{R, \sigma}^{(\nu)}\left(|h|_{\mathbb{G}}\right)
$$

where

$$
\begin{aligned}
\Phi_{R, \sigma}^{(\nu)}(\delta) & =\frac{\sigma}{R^{Q}} \int_{\frac{\delta}{\nu R}}^{1} d \tau \frac{\varphi(\sigma \tau)}{\tau^{p+Q}}=\frac{\sigma}{R^{Q}}\left(\frac{\nu R}{\delta}\right)^{p+Q-1} \int_{1}^{\frac{\nu R}{\delta}} d r \frac{\varphi\left(\frac{\sigma}{\nu R} \delta r\right)}{r^{p+Q}} \\
& \leq \frac{\sigma(\nu R)^{p+Q-1}}{R^{Q}} \frac{1}{\delta^{p}} \frac{\varphi\left(\frac{\sigma}{\nu R} \delta\right)}{\delta^{Q-1}} \int_{1}^{\infty} d r \frac{1}{r^{p+Q}} \\
& =\frac{1}{p+Q-1} \frac{\sigma(\nu R)^{p+Q-1}}{R^{Q}} \frac{1}{\delta^{p}} \frac{\varphi\left(\frac{\sigma}{\nu R} \delta\right)}{\delta^{Q-1}} .
\end{aligned}
$$


If we now set the scale $\sigma$ to $\sigma=\lambda \nu R$, we obtain

$$
\Phi_{R, \lambda \nu R}^{(\nu)}(\delta) \leq \frac{\lambda \nu^{p+Q}}{p+Q-1} R^{p} \frac{1}{\delta^{p}} \frac{\varphi(\lambda \delta)}{\delta^{Q-1}}
$$

which provides the desired result, indeed (34) becomes

$$
\begin{aligned}
I & \leq C R^{p} \int_{B_{X}\left(x_{0}, \nu R\right)} d y \int_{B_{X}(0, \nu R)} d h \frac{|f(y \cdot h)-f(y)|^{p}}{d_{X}(h, 0)^{p+Q-1}} \frac{\varphi\left(\lambda d_{X}(h, 0)\right)}{\int_{0}^{(\epsilon+2 a T) \lambda R} \varphi(\tau) d \tau} \\
& \leq C_{p, Q} R^{p} \int_{B\left(x_{0}, \lambda \nu R\right)} d y \int_{B(0, \lambda \nu R)} d h \frac{|f(y \cdot h)-f(y)|^{p}}{|h|_{\mathbb{G}}^{p+Q-1}} \frac{\varphi\left(|h|_{\mathbb{G}}\right)}{\int_{0}^{\beta R} \varphi(\tau) d \tau} .
\end{aligned}
$$

Corollary 4.6 (Poincaré-Ponce inequality). Let $\mathbb{G}$ be a Carnot group, $|\cdot|_{\mathbb{G}}$ a homogeneous norm with parameter $\lambda$ as in (6), and $\left\{\rho_{n}\right\}$ a family of radial mollifiers as in Definition 3.1 such that each $\rho_{n}$ is nonincreasing. Then for all balls $B$ of radius $R>0$ and all $C>C_{p, Q}$ there exists an $n_{0}$ such that

$$
\int_{B} d x\left|f(x)-f_{B}\right| \leq C R^{p} \int_{\mu B} d x \int_{\mu B} d y \frac{|f(x)-f(y)|^{p}}{\left|y^{-1} \cdot x\right|_{\mathbb{G}}^{p}} \rho_{n}\left(\left|y^{-1} \cdot x\right|_{\mathbb{G}}\right)
$$

for all $n>n_{0}$ and all $f$ in $L_{\text {loc }}^{p}(\mathbb{G}), 1 \leq p<\infty$, where $n_{0}$ is determined by

$$
\int_{0}^{\beta R} \rho_{n}(\tau) \tau^{Q-1} d \tau>\frac{C_{p, Q}}{C} \quad \forall n>n_{0}
$$

and constants $\mu, \beta$ are as in Theorem 4.5.

Proof. The proof follows by applying inequality (27) to a family of one-dimensional mollifiers $\left\{\rho_{n}^{(1)}\right\}$ as given by (12) in place of the function $\varphi$, with the additional requirement that each $\rho_{n}^{(1)}$ is nonincreasing, and using relation (13). This is the same as we did for Corollary 4.2

We can see that the dependence of the threshold $n_{0}$ on the dimension of the domain of evaluation is intrinsic in this approach of localization of finite differences, as already pointed out after Corollary 4.2. In particular, to get a Poincaré-like estimate, the localizing scale of the mollifiers must tighten as the balls on which the oscillations of the function are evaluated, at the left hand side, tighten.

We note in addition that, if we choose a homogeneous norm which is invariant under horizontal rotations, by the result of the previous section we can recover the classical Poincaré inequality by taking the limit $n \rightarrow \infty$.

We conclude the section extending to Carnot groups a result of [25], 3] which involves fractional Sobolev norms. Namely, we can obtain from (27) a Poincaré inequality for norms of Gagliardo type. For relationships among different fractional Sobolev norms in the Euclidean setting see for instance [1], 20] and [19].

Corollary 4.7. Let $\mathbb{G}$ be a Carnot group and $|\cdot|_{\mathbb{G}}$ a homogeneous norm with parameter $\lambda$ as in (6). Then

$$
\int_{B} d x\left|f(x)-f_{B}\right|^{p} \leq C_{p, Q, s}(1-s) p R^{s p} \int_{\mu B} d x \int_{\mu B} d y \frac{|f(y)-f(x)|^{p}}{\left|y^{-1} \cdot x\right|_{\mathbb{G}}^{Q+s p}}
$$


for all balls $B$ of radius $R$, all $f$ in $L_{l o c}^{p}(\mathbb{G}), p \geq 1$, and all $s \in\left[1-\frac{1}{p}, 1\right)$, where constants $\mu$ and $\beta$ are as in Theorem 4.5 and

$$
C_{p, Q, s}=C_{p, Q} \beta^{(1-s) p} \text {. }
$$

Proof. Let us choose a function

$$
\varphi(\tau)=\frac{\tau^{Q-1}}{\tau^{Q-(1-s) p}}=\frac{1}{\tau^{1-(1-s) p}} .
$$

If $s \geq 1-1 / p$ then $\varphi$ is nonincreasing, and if $s<1 \varphi$ is in $L_{l o c}^{1}$, so we can apply inequality (27) and the proof follows by direct computation.

Inequality (35) possesses a self improving property which can be exploited by making use of the very general results contained in [11. Moreover, by making use of a technique described in [10, it is possible to strengthen the inequality with a reduction of the domain of integration on the right, which we perform at first.

We recall here the content of these two results, restricted to the case of interest for our applications. First, we state Theorem 5.2 of [10], which consists of an application of the Boman chain technique.

Definition 4.8 (Boman chain condition). We say that a domain $\Omega$ satisfies a Boman chain condition $\mathcal{F}(\tau, M)$ for some $\tau \geq 1$ and $M \geq 1$ if there exists a covering $W$ of $\Omega$ consisting of balls $B$ such that

(1) $\tau$-dilated balls have $M$-finite overlapping: $\sum_{B \in W} \chi_{\tau B}(x) \leq M \chi_{\Omega}(x)$;

(2) there is a central ball $B^{*} \in W$ such that for all balls $B \in W$ there exists a finite chain of balls $\left\{B_{j}\right\}_{j=1}^{l(B)}$, with $B_{1}=B^{*}$ and $B_{l(B)}=B$ such that

(i) $B \subset M B_{j}$;

(ii) there exists a family of balls $\left\{R_{j}\right\}_{j=2}^{l(B)}$ such that $R_{j} \subset B_{j} \cap B_{j-1}$ and $B_{j} \cup B_{j-1} \subset M R_{j}$.

The notions of Boman domain and John domain are intimately connected, and in particular Carnot-Carathéodory balls are Boman domains (see [5]).

Theorem 4.9 (Franchi, Gutiérrez, Wheeden). Let $(X, d, \mu)$ be a quasimetric space with $\mu$ doubling, $\Omega \subset X$ a domain satisfying a Boman chain condition $\mathcal{F}(\tau, M)$, and $f i x 1 \leq p \leq q<\infty$. If $f$ and $g$ are measurable functions on $\Omega$ such that for any ball $B$ with $\tau B \subset \Omega$ it holds

$$
\left\|f-f_{B}\right\|_{L^{q}(B)} \leq A\|g\|_{L^{p}(\tau B)}
$$

with $A$ independent on $B$, then there exists a constant $c_{0}=c_{0}(\tau, M, q)$ such that

$$
\left\|f-f_{B}\right\|_{L^{q}(\Omega)} \leq c A\|g\|_{L^{p}(\Omega)} .
$$

If we apply this result to Corollary (4.7) we get the following

Corollary 4.10. Let $\mathbb{G}$ be a Carnot group, then

$$
\int_{B_{X}} d x\left|f(x)-f_{B_{X}}\right|^{p} \leq C(1-s) p R^{s p} \int_{B_{X}} d x \int_{B_{X}} d y \frac{|f(y)-f(x)|^{p}}{d_{X}\left(y^{-1} \cdot x\right)^{Q+s p}}
$$


for all $C C$ balls $B_{X}$ of radius $R$, all $f$ in $L_{\text {loc }}^{p}(\mathbb{G}), 1 \leq p<\infty$, and all $s \in\left[1-\frac{1}{p}, 1\right)$, where constants $\mu$ and $\beta$ are as in Theorem 4.5 and

$$
C=c_{0}^{2} C_{p, Q} \beta^{(1-s) p} \mu^{-s p}
$$

with $c_{0}$ coming from Theorem 4.9. All constants are intended with $\lambda=1$.

Proof. If we take Corollary (4.7) with $d_{X}(x, 0)$ as homogeneous norm, we clearly have $\lambda=1$. Then, for any fixed $R>0$ and $x_{0} \in \mathbb{G}$ we have that $\Omega=B_{X}\left(x_{0}, R\right)$ is a Boman domain for all $\tau$, and in particular for $\tau=\mu$. If we then take balls $B_{j}=B_{X}\left(x_{j}, r_{j}\right)$ such that $\mu B_{j} \subset \Omega$, we have $r_{j}<\frac{R}{\mu}$, since the diameter of $\mathrm{CC}$ balls of radius $r$ is exactly $2 r$. Then inequality (35) for the family $\left\{B_{j}\right\}$ reads

$$
\int_{B_{j}} d x\left|f(x)-f_{B_{X}}\right|^{p} \leq A \int_{\mu B_{j}} d x \int_{\mu B_{j}} d y \frac{|f(y)-f(x)|^{p}}{d_{X}\left(y^{-1} \cdot x\right)^{Q+s p}}
$$

with $A=C_{p, Q, s}(1-s) p\left(\frac{R}{\mu}\right)^{s p}$, which does not depend on $B_{j}$.

We now apply Theorem 4.9 twice: the first time to $g_{1}(x)=\|u(x, .)\|_{L^{p}\left(\mu B_{j}\right)}$ and the second time to $g_{2}(x)=\|u(., y)\|_{L^{p}\left(B_{j}\right)}$, where $u(x, y)$ stands for $u(x, y)=\frac{|f(y)-f(x)|^{p}}{d_{X}\left(y^{-1} \cdot x\right)^{Q+s p}}$.

We recall now Theorem 2.3, Corollary 2.4 and Remark 2.6 of [11].

Theorem 4.11 (Franchi, Pérez, Wheeden). Let $(X, d, \mu)$ be a metric measure space such that the distance $d$ has the segment property, i.e. for all $x, y \in X$ there exists a continuous curve $\gamma:[0, L] \rightarrow X$ joining $x$ to $y$ such that $d(\gamma(t), \gamma(s))=|t-s|$ for all $0 \leq t, s \leq L$, and let $\mu$ be a doubling measure, i.e. there exists a constant $C_{d}$ such that

$$
0<\mu(B(x, 2 r)) \leq C_{d} \mu(B(x, r))<\infty
$$

for all $x \in X$ and all $r>0$, where $B$ stands for the open ball with respect to d. We will denote by $\mathcal{B}$ the class of such balls.

Suppose that

i) $a: \mathcal{B} \rightarrow \mathbb{R}^{+}$is a functional on balls for which there exists a constant $c<\infty$ and a constant $1 \leq r<\infty$ such that it holds

$$
\sum_{i} a\left(B_{i}\right)^{r} \mu\left(B_{i}\right) \leq c^{r} a(B)^{r} \mu(B)
$$

for any ball $B$ and any family $\left\{B_{i}\right\}$ of subballs of $B$ with bounded overlaps, that is there exist an integer $M \geq 0$ such that for each $B_{i}$ there are at most $M$ other balls of the same family intersecting it;

ii) $f: B_{0} \rightarrow \mathbb{R}$ is a function, defined on a ball $B_{0}$, satisfying

$$
f_{B}\left|f-f_{B}\right| d \mu \leq a(B)
$$

for any ball $B \subset B_{0}$. 
Then there exists a constant $c^{\prime}$ independent of $f$ and $B_{0}$ such that

$$
\left(f_{B_{0}}\left|f-f_{B_{0}}\right|^{p} d \mu\right)^{1 / p} \leq c^{\prime}\|a\| a\left(B_{0}\right)
$$

for any $1<p<r$, where we have denoted by $\|a\|$ the smallest constant $c$ such that (37) holds.

Corollary 4.12. Let $\mathbb{G}$ be a Carnot group, then

$$
\left(f_{B_{X}} d x\left|f(x)-f_{B_{X}}\right|^{p}\right)^{1 / p} \leq C(1-s) R^{Q+s} f_{B_{X}} d x f_{B_{X}} d y \frac{|f(x)-f(y)|}{\left|y^{-1} \cdot x\right|_{\mathbb{G}}^{Q+s}}
$$

for all $C C$ balls $B_{X}$ of radius $R$, all $s \in(0,1)$, all $f \in L_{\text {loc }}^{1}(\mathbb{G})$ and all $1 \leq p<p^{*}$, where the critical exponent is given by $p^{*}=\frac{Q}{Q-s}$.

Proof. Inequality (4.12) is a direct consequence of Theorem 4.11 applied to Corollary 4.10. If we take $p=1$ in (36) we get

$f_{B_{X}\left(x_{0}, R\right)} d x\left|f(x)-f_{B_{X}}\right| \leq C(1-s) R^{s-Q} \int_{B_{X}\left(x_{0}, R\right)} d x \int_{B_{X}\left(x_{0}, R\right)} d y \frac{|f(x)-f(y)|}{d_{X}\left(y^{-1} \cdot x\right)^{Q+s}}$

The requirement of having balls satisfying the segment property is attained for geodesic balls, and the doubling condition is satisfied by homogeneity, so we need only to verify condition (37) for the functional

$$
a\left(B_{X}\left(x_{0}, R\right)\right)=R^{s-Q} \int_{B_{X}\left(x_{0}, R\right)} d x \int_{B_{X}\left(x_{0}, R\right)} d y g(x, y)
$$

where

$$
g(x, y)=\frac{|f(x)-f(y)|}{d_{X}\left(y^{-1} \cdot x\right)^{Q+s}}
$$

in order to do that, let us take a family $\left\{B_{i}\right\}$ of $M$-finite overlapping balls $B_{i} \subset B_{X}=B_{X}\left(x_{0}, R\right)$ of radius $R_{i}$. Then

$$
\begin{aligned}
\sum_{i} a\left(B_{i}\right)^{r} R_{i}^{Q} & =\sum_{i}\left(\int_{B_{i}} d x \int_{B_{i}} d y g(x, y)\right)^{r} R_{i}^{(s-Q) r+Q} \\
& \leq R^{(s-Q) r+Q} \sum_{i}\left(\int_{B_{X}} d x \int_{B_{X}} d y g(x, y) \chi_{B_{i}}(x) \chi_{B_{i}}(y)\right)^{r} \\
& \leq R^{(s-Q) r+Q}\left(\sum_{i} \int_{B_{X}} d x \int_{B_{X}} d y g(x, y) \chi_{B_{i}}(x) \chi_{B_{i}}(y)\right)^{r} \\
& \leq M^{2 r}\left(a\left(B_{X}\right)\right)^{r} R^{Q}
\end{aligned}
$$

indeed, the first inequality holds if $(s-Q) r+Q>0$, and the last one is due to the finite overlapping of the family of balls, provided $r \geq 1$.

\section{REFERENCES}

[1] R. Adams, Sobolev spaces, Academic Press, 1975.

[2] A. Bonfiglioli, E. Lanconelli and F. Uguzzoni, Statified Lie Groups and potential theory for their sub-Laplacians, Springer, 2007. 
[3] J. Bourgain, H. Brezis and P. Mironescu, Another look at Sobolev Spaces, in: J.L. Menaldi, E. Rofman and A. Sulem (Eds), Optimal Control and Partial Differential Equations (volume in Honour of A. Bensoussan's 60th birthday), IOS Press, 2001, pp. $439-455$.

[4] H. Brezis, How to recognize constant functions. Connections with Sobolev Spaces, Russian Math. Surveys 57 (2002) 693-708.

[5] S. Buckley, P. Koskela and G. Lu, Boman equals John, XVIth Rolf Nevalinna Colloquium, de Gruyter, 1996, pp. 91-99.

[6] G.B. Folland, Subelliptic estimates and function spaces on nilpotent Lie groups, Ark. Mat. 13 (1975), 161-207.

[7] G.B. Folland, E.M. Stein, Hardy spaces on homogeneous groups, Princeton University Press, 1982.

[8] B. Franchi, BV spaces and rectifiability for Carnot-Carathéodory metrics: an introduction, VII NAFSA Spring School, Math. Inst. Acad. Sc. Czech Rep., 2003, pp. $73-132$.

[9] B. Franchi, E. Lanconelli, Hölder regularity for a class of linear nonuniformly elliptic operators with measurable coefficients, Ann. Sc. Norm. Super. Pisa Cl. Sci. IV Ser. 10 (1983) 523-541.

[10] B. Franchi, C. E. Gutierrez, R. L. Wheeden, Weighted Sobolev-Poincaré inequalities for Grushin-type operators, Comm. Part. Diff. Eq. 19 (3) (1994) 523-604.

[11] B. Franchi, C. Pérez, R. L. Wheeden, Self-Improving properties of John-Nirenberg and Poincaré inequalities on spaces of homogeneous type, J. Funct. Anal. 153 (1998) 108-146.

[12] B. Franchi, R. Serapioni, and F. Serra Cassano, Rectifiability and perimeter in the Heisenberg group, Math. Ann. 321 (3) (2001) 479-531.

[13] B. Franchi, R. Serapioni, and F. Serra Cassano, Meyers-Serrin type theorems and relaxation of variational integrals depending on vector fields, Houston J. Math. 22 (4) (1996) 859-890.

[14] B. Franchi, R. Serapioni, and F. Serra Cassano, On the structure of finite perimeter sets in step 2 groups, J. Geom. An. 13 (2003) 421-466.

[15] M. Gromov, Carnot-Carathéodory spaces seen from within, Progress in Math. 144, Birkhäuser, 1996.

[16] P. Hailasz, P.Koskela, Sobolev met Poincaré, Mem. Amer. Math. Soc. 688, 2000.

[17] J. Heinonen, Lectures on analysis on metric spaces, Springer, 2001.

[18] A. W. Knapp, E. M. Stein, Interwining operators for semi-simple groups, Annals of Math. 93 (1971) 489-578.

[19] J.L. Lions, E. Magenes, Problemi ai limiti non omogenei (III), Ann. Sc. Norm. Super. Pisa Cl. Sci. III Ser. 15 (1961) 41-103.

[20] V. Maz'ya, Sobolev spaces, Springer, 1985.

[21] R. Monti, F. Serra Cassano, Surface measures in Carnot-Carathéodory spaces, Calc. Var. Part. Diff. Eq. 13 (2001) 339-376.

[22] D. Morbidelli, Functional Sobolev norms and structure of Carnot-Carathéodory spaces, Studia Math. 139 (3) (2000) 213-244.

[23] A. Nagel, E.M. Stein, S. Wainger, Balls and metrics defined by vector fields I: Basic properties, Acta Math. 155 (1985) 103-147.

[24] P. Pansu, Métriques de Carnot-Carathéodory et quasiisométries des espaces symmétriques de rang un, Annals of Math. 129 (1989) 1-60.

[25] A. Ponce, An estimate in the spirit of the Poincaré's inequality, J. Eur. Math. Soc. 6 (2004) 1-15.

[26] E.M. Stein, Harmonic analysis: real-variable methods, orthogonality and oscillatory integrals, Princeton University Press, 1993.

[27] V.S.Varadarajan, Lie groups, Lie algebras and their representations, Springer, 1984.

[28] N.Th. Varopoulos, L. Saloff-Coste and T. Coulhon, Analysis and geometry on groups, Cambridge University Press, 1992.

[29] N.Th. Varopoulos, Fonctions harmoniques sur les groupes de Lie, C. R. Acad. Sci. Paris Sér. I Math. 304 (17) (1987) 519-521. 